\title{
Single-Channel Behavior of Heteromeric $\alpha 1 \beta$ Glycine Receptors: An Attempt to Detect a Conformational Change before the Channel Opens
}

\author{
Valeria Burzomato, Marco Beato, Paul J. Groot-Kormelink, David Colquhoun, and Lucia G. Sivilotti \\ Department of Pharmacology, University College London, London WC1E 6BT, United Kingdom
}

The $\alpha 1 \beta$ heteromeric receptors are likely to be the predominant synaptic form of glycine receptors in the adult. Their activation mechanism was investigated by fitting putative mechanisms to single-channel recordings obtained at four glycine concentrations (10-1000 $\mu \mathrm{M})$ from rat $\alpha 1 \beta$ receptors, expressed in human embryonic kidney 293 cells. The adequacy of each mechanism, with its fitted rate constants, was assessed by comparing experimental dwell time distributions, open-shut correlations, and the concentration-open probability $\left(P_{\text {open }}\right)$ curve with the predictions of the model. A good description was obtained only if the mechanism had three glycine binding sites, allowed both partially and fully liganded openings, and predicted the presence of open-shut correlations. A strong feature of the data was the appearance of an increase in binding affinity as more glycine molecules bind, before the channel opens. One interpretation of this positive binding cooperativity is that binding sites interact, each site sensing the state of ligation of the others. An alternative, and novel, explanation is that agonist binding stabilizes a higher affinity form of the receptor that is produced by a conformational change ("flip") that is separate from, and precedes, channel opening. Both the "interaction" scheme and the flip scheme describe our data well, but the latter has fewer free parameters and above all it offers a mechanism for the affinity increase. Distinguishing between the two mechanisms will be important for our understanding of the structural dynamics of activation in the nicotinic superfamily and is important for our understanding of mutations in these receptors.

Key words: glycine; binding; gating; single channel; dose-response; patch clamp; kinetics

\section{Introduction}

For ligand-gated ion channels, the big question, at the molecular level, is how binding of an agonist causes the channel to open. The binding site for the agonist is a long way from the channel gate

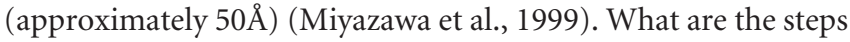
that make up this reaction? How are these steps connected? Which steps involve binding and which are conformation changes? How fast is each step? Why are partial agonists partial? Only by answering these questions can we achieve the aim of relating function to structure.

For channels in the nicotinic superfamily, extensive singlechannel work, particularly on the muscle-type nicotinic acetylcholine receptor itself, has established some key facts about the activation process, in terms of both function (Colquhoun and Sakmann, 1985; Sine et al., 1990; Grosman et al., 2000) and structure (Brejc et al., 2001; Unwin et al., 2002; Miyazawa et al., 2003; Celie et al., 2004). Changes at the binding site precede channel

Received Aug. 19, 2004; revised 0ct. 18, 2004; accepted 0ct. 18, 2004.

This work was supported by Medical Research Council grants (D.C., L.G.S.), a Special Research Training Fellowship in Bioinformatics/Neuroinformatics (M.B.), and by Wellcome Trust Grant 064652 (L.G.S.). V.B. was supported by the School of Pharmacy (Millennium Studentship) and by Fondazione Gini (Padua, Italy). We are grateful to Prof. Alan Hawkes, Prof. Frank Ball, and Dr. Andrew Plested for critical discussion.

Correspondence should be addressed to Lucia Sivilotti, Medical Sciences Building, University College London, Gower Street, London WC1E 6BT, UK. E-mail: I.sivilotti@ucl.ac.uk.

DOI:10.1523/JNEUROSCI.3424-04.2004

Copyright $\odot 2004$ Society for Neuroscience $\quad 0270-6474 / 04 / 2410924-17 \$ 15.00 / 0$ opening, and the two may be linked by a wave of conformational changes in which successive discrete subdomains between the binding site and the pore move in a sequence of rigid body movements (Chakrapani et al., 2004). For both nicotinic (Lester et al., 2004) and glutamate (Gouaux, 2004) receptors, structural changes that occur after agonist binding have been described, although only Torpedo channels have been imaged in both the closed and (at lower resolution) open (Unwin, 1995) state. The goal of understanding gating as a dynamic change in structure remains unattained.

The structural studies suggest that binding of agonist causes closure of the molecule around the bound ligand (which, presumably, results in an increased affinity). One of the outstanding questions is how this domain closure is related to the opening of the channel.

In this study we extend our recent work on the homomeric glycine receptor, another member of the nicotinic superfamily (Beato et al., 2004), to an investigation of the main synaptic form of glycine receptors in the adult CNS, the heteromeric $\alpha 1 \beta$ channel. Direct fitting of activation mechanisms to single channel data shows that conventional mechanisms can describe channel behavior only if it is postulated that the affinity for binding of glycine to the shut state increases when other binding sites have already been occupied by glycine. This suggests that binding sites interact while still shut, and in view of the large separation between the sites, this in turn suggests that a substantial conforma- 
tion change is caused by binding while the channel is still shut. A mechanism that postulates such a pre-opening conformation change fits the data well (although not uniquely) and provides an explanation for the apparent "cooperativity" of binding along exactly the same lines as first proposed by Wyman and Allen (1951) for the apparent cooperativity of binding of oxygen to hemoglobin (Colquhoun, 1998).

\section{Materials and Methods}

Heterologous expression of rat $\alpha 1 \beta$ glycine receptors in human embryonic kidney 293 cells. Human embryonic kidney 293 cells (HEK293) (American Type Culture Collection-CRL-1573; LGC Promochen, Teddington, UK) were maintained at $37^{\circ} \mathrm{C}$ in a $95 \%$ air $/ 5 \% \mathrm{CO}_{2}$ incubator in DMEM supplemented with $0.11 \mathrm{~g} / \mathrm{l}$ sodium pyruvate, $10 \% \mathrm{v} / \mathrm{v}$ heat-inactivated fetal bovine serum, $100 \mathrm{U} / \mathrm{ml}$ penicillin $\mathrm{G}, 100 \mu \mathrm{g} / \mathrm{ml}$ streptomycin sulfate, and $2 \mathrm{~mm}$ L-glutamine (all from Invitrogen, Paisley, UK) and passaged every $2-3 \mathrm{~d}$, up to 20 times.

Cells were plated on dishes, incubated for $10 \mathrm{hr}$, and then transfected by a calcium phosphate-DNA coprecipitation method (GrootKormelink et al., 2002) with cDNAs for the rat $\alpha 1$ and $\beta$ glycine receptor subunits. For the amplification and cloning of the rat $\alpha 1$ (GenBank accession number AJ310834) and $\beta$ (GenBank accession number AJ310839) GlyR subunits into the pcDNA3.1(+) vector (Invitrogen, Breda, The Netherlands) see Beato et al. (2002) and Burzomato et al. (2003), respectively. The $\beta$ subunit plasmid was transfected in a 40 or $20 \times$ greater quantity than the $\alpha 1$ plasmid to minimize contamination by homomeric $\alpha 1$ receptors. In all cases the marker enhanced green fluorescent protein plasmid (EGFP-c1) (BD Biosciences, Oxford, UK) was cotransfected (18\% and $37 \%$ of the total plasmid DNA for the 1:40 and 1:20 transfections, respectively) to allow detection of transfected cells. Each dish was transfected with $3 \mu \mathrm{g}$ of DNA. Patch-clamp recordings were made 14-48 hr after transfection.

Spinal cord slices. Acute spinal cord slices (350 $\mu \mathrm{m}$ thickness) were obtained from postnatal day 17 (P17)-P18 rats (Takahashi et al., 1992). The animals were anesthetized with an intraperitoneal injection of urethane [10\% (w/v) solution; Sigma-Aldrich, Gillingham, UK) and decapitated. The spinal cord was exposed after ventral laminectomy. The midthoracic to lumbar region was removed and fixed vertically to an agar block using tissue glue (Vetbond, World Precision Instruments, Hertfordshire, UK). The block was glued to the base of the slicing chamber of a Leica VT1000 Vibratome, and 8-10 slices were taken from the lumbar region spanning the L2-L5 segments. After $30 \mathrm{~min}$ of incubation at $37^{\circ} \mathrm{C}$, slices were left at room temperature for another $30 \mathrm{~min}$ and then transferred to the recording chamber as needed. Slices were bubbled continuously with $95 \% \mathrm{O}_{2} / 5 \% \mathrm{CO}_{2}$.

Electrophysiological recording. All single-channel recordings from transfected cells were obtained in the cell-attached configuration; a total of 21 patches were selected for analysis. Cells were bathed in an extracellular solution containing (in mM): $102.7 \mathrm{NaCl}, 20 \mathrm{Na}$ gluconate, $4.7 \mathrm{KCl}$, $2 \mathrm{CaCl}_{2}, 1.2 \mathrm{MgCl}_{2}, 10$ HEPES, 14 glucose, 15 sucrose, 20 TEACl, $\mathrm{pH}$ adjusted to 7.4 with $\mathrm{NaOH}$; osmolarity, $320 \mathrm{mOsm}$. HPLC-grade water was used to prepare the solution to minimize contamination of glycine.

Extracellular solutions for dissecting and recording from acute spinal cord slices had the same composition (in $\mathrm{mm}$ ): $113 \mathrm{NaCl}, 3 \mathrm{KCl}, 25$ $\mathrm{NaHCO}_{3}, 1 \mathrm{NaH}_{2} \mathrm{PO}_{4}, 2 \mathrm{CaCl}_{2}, 2 \mathrm{MgCl}_{2}, 11$ D-glucose, $\mathrm{pH}$ 7.4. The intracellular solution for whole-cell recordings consisted of (in $\mathrm{mM}$ ): 140 $\mathrm{CsCl}, 4 \mathrm{NaCl}, 1 \mathrm{Mg}_{2} \mathrm{Cl}, 0.5 \mathrm{CaCl}_{2}$, 5 EGTA, 10 HEPES, 2 Mg-ATP, pH 7.3 with $\mathrm{CsOH}$.

Patch pipettes for cell-attached single-channel recording were pulled from thick-walled borosilicate glass (GC150F; Harvard Apparatus, Edenbridge, UK), coated with Sylgard (Dow Corning, Coventry, UK), firepolished to a resistance of 10-15 M $\Omega$, and filled with extracellular solution containing $10-1000 \mu \mathrm{M}$ glycine. Cell-attached patches were voltage clamped at $+100 \mathrm{mV}$, and single-channel currents were recorded with an Axopatch 200B amplifier, prefiltered at $10 \mathrm{kHz}$ with the amplifier fourpole Bessel filter, and stored on digital audio tape (Bio-Logic Science
Instruments, Claix, France). Recordings were replayed from the tape, filtered at $3 \mathrm{kHz}$ with a eight-pole Bessel filter, and digitized at $30 \mathrm{kHz}$ (Digidata 1322A; Molecular Devices, Wokingham, UK; Clampex software) for off-line analysis.

We discarded all patches in which homomeric channels were detected, even if homomeric openings were few. For openings that are long enough to reach full amplitude, homomeric openings are easily distinguishable from heteromeric ones on the basis of their conductances (for review, see Legendre, 2001). At a holding potential of $+100 \mathrm{mV}$ (no junction potential correction), the channel amplitude was $4.7 \pm 0.1$ and $3.1 \pm 0.1 \mathrm{pA}$ for homomeric and heteromeric channels, respectively (Burzomato et al., 2003; Beato et al., 2004).

Recordings from acute spinal cord slices were performed from ventral horn neurons visually identified as motoneurons on the basis of their location, size, and shape, with an infrared-differential interference contrast microscope. Microelectrodes were pulled from thick-walled borosilicate glass (GC150F; Harvard Apparatus) to 1-1.5 M $\Omega$ resistance and fire polished to a final resistance of approximately $2.5 \mathrm{M} \Omega$. Cells were voltage clamped at $-70 \mathrm{mV}$, and only those cells with stable holding current (less than $40 \mathrm{pA}$ ) for the duration of the experiment were included for analysis. Series resistance (6-10 M 2 ) was compensated (70$90 \%$ ). To isolate glycinergic miniature IPSCs (mIPSCs), $0.5 \mu \mathrm{M}$ tetrodotoxin, $5 \mu \mathrm{M}$ SR95531 hydrobromide, $20 \mu \mathrm{M}$ AP5, and $10 \mu \mathrm{M}$ CNQX were added to the recording solution to block sodium action potentials, $\mathrm{GABA}_{\mathrm{A}}, \mathrm{NMDA}$, and AMPA-kainate receptors, respectively.

Analysis of single-channel recordings. Recordings were idealized by time-course fitting with the program SCAN (Colquhoun and Sigworth, 1995). This produced a list of openings and shuttings together with the (fitted) amplitude values for each opening. All programs used in our analysis can be obtained from http://www.ucl.ac.uk/pharmacology/ dc.html.

The first stage of analysis was to construct distributions of fitted amplitudes, shut times, and open periods from the experimental data. These distributions were fitted (EKDIST program) by a single Gaussian for the amplitude histogram and by a mixture of exponential densities for dwell times. Only openings longer than twice the rise time of the filter contributed to the fitted amplitudes histogram. A time resolution of $30 \mu \mathrm{s}$ (for both open and shut times) was imposed retrospectively (Colquhoun and Sigworth, 1995). This stage of fitting is purely descriptive and empirical: the parameters so estimated (number of components, time constants, areas; see Table 1) were not used in the subsequent stage (the testing of mechanisms; see below). These fits were needed only to choose the critical shut time $\left(t_{\text {crit }}\right)$ value that was used to divide recordings into sections (bursts or clusters) that are believed to originate from one individual channel. At low agonist concentrations, the groups will be individual channel activations.

Single-channel open probability-concentration curve. At glycine concentrations $>10 \mu \mathrm{M}$, channel openings occurred in clusters separated by long quiescent periods, probably reflecting sojourns in long-lived desensitized states (Sakmann et al., 1980; Colquhoun and Ogden, 1988). Only clusters that contained more than 5 openings and no double openings were selected for further analysis (17-91 clusters analyzed per concentration; clusters contained up to 3147 openings; mean 340 openings). Each of these clusters is likely to represent the activity of a single glycine channel (see Results).

For each patch (three to four per concentration; see Table 2) the open probability $\left(P_{\text {open }}\right)$ was estimated as the ratio between the sum of the open times within clusters and the sum of the durations of the clusters (both obtained from the idealized recordings). This procedure effectively weights the contribution of each cluster to the $P_{\text {open }}$ value according to their duration, because $P_{\text {open }}$ estimates derived from longer clusters are more precise.

The $P_{\text {open }}$ values obtained for patches at the same concentration were then averaged (see Table 2). The $P_{\text {open }}$-concentration curve described by these points was fitted empirically with the Hill equation (weighted least squares fit) using the CVFIT program to estimate $I_{\max }, E C_{50}$, and the Hill slope. This Hill slope was compared with that predicted by fitted mech- 
anisms. The latter will not have constant Hill slopes, so the Hill slope at $E C_{50}$, defined as follows, was found numerically:

$$
\left.n_{\mathrm{H} 50} \equiv \frac{\mathrm{d} \ln \left(\frac{P_{\text {open }}}{P_{\max }-P_{\text {open }}}\right)}{\mathrm{d} \ln (G)}\right|_{G=E C_{50}},
$$

where $G$ is the glycine concentration. For this calculation we used the apparent $P_{\text {open }}$ value (that expected in the presence of missed events), which was calculated using Equations 2-4.

Fitting alternative mechanisms to single-channel data with HJCFIT. Maximum likelihood fittings for the evaluation of kinetic schemes and rate constants were performed with the HJCFIT program (Colquhoun et al., 1996, 2003). The experimental input into this program consists of one or more idealized recordings, i.e., the entire sequence of durations of open and shut times in the order in which they occur, together with the imposed resolution. Recordings were obtained at four glycine concentrations $(10,30,100$, and $1000 \mu \mathrm{M})$; these produced an apparent $P_{\text {open }}$ of $8-98 \%$. Three recordings at each concentration, each $12,000-19,000$ transitions long, were then selected on the basis of signal-to-noise ratio. These recordings were then arranged in three independent sets; each set consisted of four experiments, one at each of the four concentrations. All experiments in a set were fitted simultaneously with HJCFIT.

From the data, the resolution, and a postulated mechanism, HJCFIT calculates a likelihood value for the experimental record(s) for a set of guesses for the values of the rate constants. The rate constants are then adjusted iteratively to maximize this likelihood. The imposed resolution (30 $\mu$ s in our experiments) is taken into account by implementing the exact solution for missed events correction by Hawkes et al. (1990, 1992): hence the acronym HJC (Hawkes, Jalali, and Calquhoun).

Openings were divided into groups using a critical shut time $\left(t_{\text {crit }}\right)$ so that openings within each group are likely to come from the same channel (see Results). At low agonist concentrations (10 $\mu \mathrm{M}$ glycine), the groups correspond to individual activations of the channel consisting of a few openings: the likelihood of each such group was calculated using the initial and final vectors defined by Colquhoun et al. (1996, their equations 5.8-5.11), which we refer to as CHS vectors (Colquhoun, Hawkes, and Srodzinski). We cannot interpret shut times between these groups of openings (because they depend on the number of channels in the patch, which is unknown), but CHS vectors exploit the knowledge that the true shut time between one group and the next must be longer than $t_{\text {crit }}$. At higher concentrations, at which desensitization is relevant, $\mathrm{CHS}$ vectors cannot be used because desensitized states are not included in the mechanism being fitted. We therefore used steady-state initial vectors. This is an approximation but one that has little effect, because at these concentrations the number of events in each group (cluster) is large, and this reduces the effect of the initial vector, as shown by the results of simulations (Colquhoun et al., 2003).

Each fit was repeated using several different initial guesses. If the likelihood surface has a single well defined maximum, the same rate constant estimates should be obtained no matter what initial guesses were used.

To test whether a postulated mechanism describes adequately the behavior of the receptor, the predictions of the mechanism together with the rate constants estimated by HJCFIT were compared with the experimental observations. This was done routinely by inspection of four types of data display, namely, the distributions of observed open time and shut time, conditional mean open time plots, and the $P_{\text {open }}-$ concentration curve.

The rate constants estimated for the specified mechanism, data, and resolution were used to calculate the distributions of the observed or apparent open times and shut times (HJC distributions). Observed open times, for example, are extended by missed shut times (those below the specified resolution). The appropriate HJC distribution (solid lines in the dwell-times plots; see for example the first and second rows of plots in Figure 4) is superimposed on (not fitted to) the histogram of observed values, and insofar as it fits, the proposed mechanism and the values of the rate constants are a good description of the data. The predicted distribution at perfect resolution is also plotted, as a dashed line (see Fig. 4, first and second rows of plots). This was calculated from the fitted rate constants as described by Colquhoun and Hawkes (1982), and the separation between this and the solid line shows the extent to which failure to detect short events affected the observations.

As for other channels, short open times tend to occur adjacent to long shut times. To determine whether these correlations (Fredkin et al., 1985; Colquhoun and Hawkes, 1987; Blatz and Magleby, 1989) are predicted accurately, the HJC joint (bivariate), or conditional, distributions of open time and adjacent shut time can be checked. For a synoptic view of these, we show here only conditional mean plots. In this plot, the mean of all apparent open times that are adjacent to apparent shut times in a specified range is plotted against the mean of the shut times in that range. The downward slope in this graph (see Fig. 4, third row of plots) shows that the durations of open times and adjacent shut times are negatively correlated. The HJC prediction for the conditional mean open time, calculated for the same ranges of shut times as observed values, are plotted as open circles; these are calculated as described in Colquhoun et al. (1996, section 4). Because the mechanism obeys microscopic reversibility, the predictions are the same whether we look at the preceding shut time, the following shut time, or both. The dashed line on these plots shows the theoretical continuous relationship between mean open time and adjacent shut time that would be approached if sufficiently narrow shut time ranges could be used, calculated as in Colquhoun et al. (1996, section 3).

One of the criteria used to judge how well a postulated mechanism described the data was to see how well the equilibrium concentrationresponse curve was predicted, the response being the probability of a channel being open, $P_{\text {open }}$, obtained from single-channel measurements. Although $P_{\text {open }}$ is much less sensitive to missed brief events than the mean open and shut times, the correction for missed events was nevertheless found to be essential, so the observed $P_{\text {open }}$ values were compared with the predicted apparent $P_{\text {open }}$. This is not given explicitly in Colquhoun et al. (1996), so the method will be given here. The apparent $P_{\text {open }}$ is calculated as follows:

$$
P_{\mathrm{open}}^{\mathrm{HJC}}=\frac{{ }^{e} \mu_{\mathrm{open}}}{{ }^{e} \mu_{\mathrm{open}}+{ }^{e} \mu_{\text {shut }}},
$$

where ${ }^{e} \mu_{\text {open }}$ is the apparent, or extended, mean open time (extended by missed shuttings). The probability density function of apparent open time is from Colquhoun et al. (1996, Eq. 2.14) as follows:

$$
f_{\mathrm{T}}=\boldsymbol{\varphi}_{\mathrm{A}}{ }^{e} \boldsymbol{G}_{\mathrm{AF}}(t) \boldsymbol{u}_{\mathrm{F}}=\varphi_{\mathrm{A}}{ }^{\mathrm{A}} \boldsymbol{R}\left(t-t_{\mathrm{res}}\right) \boldsymbol{Q}_{\mathrm{AF}} \exp \left(\boldsymbol{Q}_{\mathrm{FF}} t_{\mathrm{res}}\right) \boldsymbol{u}_{\mathrm{F}},
$$

where the matrices are defined in Colquhoun et al. (1996, Eq. 2.1, 2.10, and 2.12). The mean can be found in general by differentiating the Laplace transform of a probability density function, changing the sign, and setting the Laplace variable, $s$, to zero. The apparent open time is therefore as follows:

$$
{ }^{e} \mu_{\text {open }}=t_{\text {res }}+\varphi_{\mathrm{A}}\left[-\frac{\mathrm{d}^{\mathrm{A}} \boldsymbol{R}^{\star}(s)}{\mathrm{d} s}\right]_{s=0} \boldsymbol{Q}_{\mathrm{AF}} \exp \left(\boldsymbol{Q}_{\mathrm{FF}} t_{\mathrm{res}}\right) \boldsymbol{u}_{\mathrm{F}} .
$$

The expression for the derivative is given in Colquhoun et al. (1996, Eq. 3.6). An analogous expression gives the mean apparent shut time. These quantities are evaluated in the HJCFIT program.

The predicted apparent $P_{\text {open }}$-concentration curve (see Fig. 4C, solid line), calculated as just described, was superimposed on (not fitted to) the observed points. For comparison, the ideal (no missed events) $P_{\text {open }}{ }^{-}$ concentration curve is also shown (dashed line). The quality of the fit was judged by eye and by comparing predicted and observed values for the $E C_{50}$ value and for the Hill slope at the $E C_{50}$.

Analysis of mIPSCs. The analysis of mIPSCs was performed on nine cells. A variable number of events (range, 120-700) were detected using Clampfit 9.2 software (Axon Instruments). The events were inspected, and all mIPSCs that were separated by less than $100 \mathrm{~ms}$ were excluded from analysis. Most IPSCs (72\%) had 20-80\% rise time lower than 0.6 $\mathrm{ms}$, indicating a majority of somatic or near-somatic locations for the glycinergic synaptic inputs to motoneurons. To avoid distortions in the estimates of amplitude and decay time, events with rise times greater than $0.6 \mathrm{~ms}$ were not included in the analysis. No correlations were detected between rise time and amplitudes or rise time and decay time, with values 


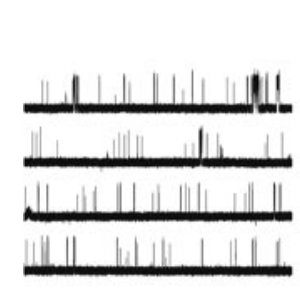

\section{$10 \mu \mathrm{M}$ glycine}

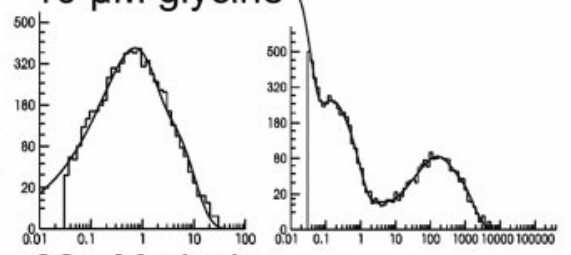

$30 \mu \mathrm{M}$ glycine
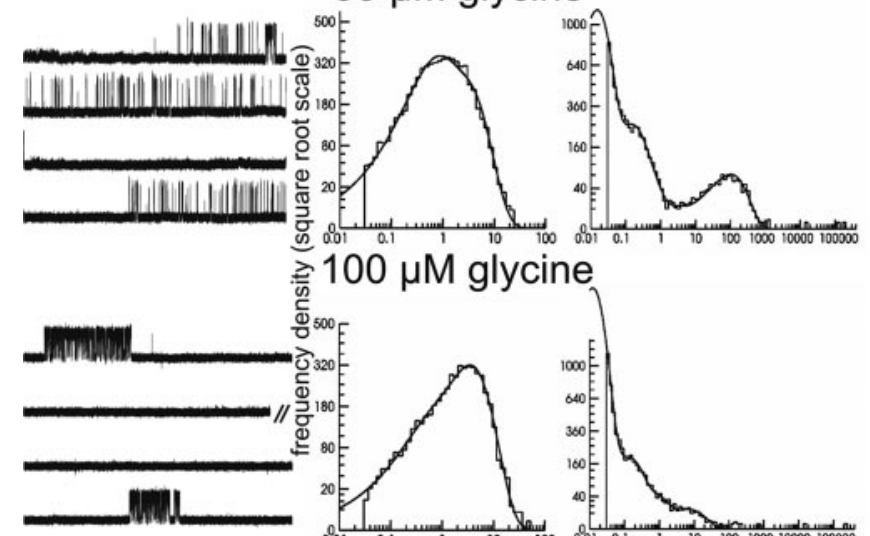

$100 \mu \mathrm{M}$ glycine
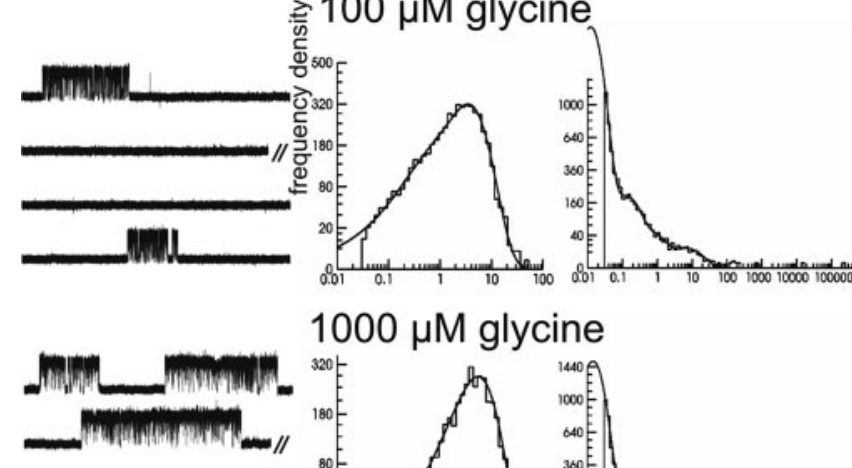

\section{$1000 \mu \mathrm{M}$ glycine}
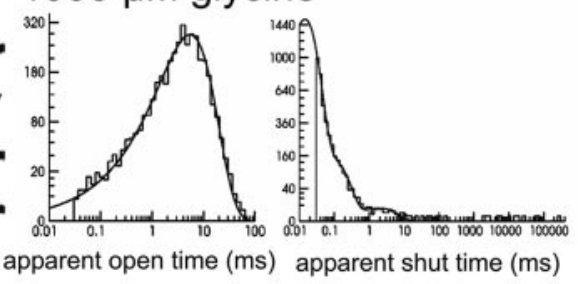

Figure 1. Activation of heteromeric $\alpha 1 \beta$ channels by increasing concentrations of glycine. The left column shows continuous sweeps of cell-attached recordings at 10, 30, 100, and 1000 $\mu \mathrm{m}$ glycine ( $3 \mathrm{kHz}$ filter). Bursts of openings are observed at $10 \mu \mathrm{m}$, whereas at higher agonist concentrations bursts group into clusters. The middle and right columns show the dwell-time distributions (apparent open and shut times) corresponding to the same patches for which traces are shown to the left. Distributions were fitted with a mixture of exponential probability density functions (Table 1).

for the Pearson correlation coefficient, $r$, in the range $-0.05-0.08$ ( $P=$ $0.7-0.9)$ and $-0.01-0.03$ ( $P=0.7-0.9)$, respectively. After filtering at 3 $\mathrm{kHz}$, the selected mIPSCs for each experiment were averaged and fitted with a single or double exponential from $90 \%$ of the peak to baseline.

All data are expressed as mean $\pm \mathrm{SD}$ of the mean. For estimated rate constants, we report the average of the estimates obtained from different sets and the coefficient of variation (CV) of the mean. Equilibrium constants were calculated separately for each set and then averaged. This is the sensible thing to do when there is a strong positive correlation between the estimates of the rate constants in the numerator and denominator (as for $E_{3}=\beta_{3} / \alpha_{3}$ ), but it means that the mean values for equilibrium constants given in the tables are not identical with the ratio of the mean rate constants in the same table.

\section{Results}

\section{Experimental dwell-time distributions}

The cell-attached recordings on the left of Figure 1 show singlechannel currents evoked by four glycine concentrations. At the lowest concentration, $10 \mu \mathrm{M}$ glycine, sparse openings occurred in groups (bursts), each of which represents a single activation of the channel. At higher concentrations there are long clusters of closely spaced openings, separated by long shut periods. These four agonist concentrations are the ones selected for the fits of mechanisms (see below). Figure 1, middle and right columns, shows the corresponding distributions of apparent open and shut times, fitted by mixtures of exponential probability density functions. The results of these empirical fits (Table 1) were used only to choose the $t_{\text {crit }}$ for the second stage of analysis (see Materials and Methods), as follows.

At the lowest concentration, $10 \mu \mathrm{M}$ glycine, the shut time distribution was fitted with six exponential components (Fig. 1, Table 1). The four fastest time constants were classified as being within-bursts if they were similar across different patches at the same concentration and across different concentrations (Table 1). Thus, a $t_{\text {crit }}$ value of $4 \mathrm{~ms}$ was chosen between the fourth and fifth shut time component, using the criterion of minimizing the total number of misclassified events (Jackson et al., 1983). The progressive shortening at higher glycine concentrations of the longer (interburst) shut times is clearly visible in the distributions in Figure 1, right-hand column, and in the expanded traces shown in Figure 2 $(A-E)$. The components become progressively harder to distinguish until, at $1000 \mu \mathrm{M}$, only three components can be resolved, although the real number of components cannot change.

At concentrations higher than $10 \mu \mathrm{M}$, activations are closely spaced and separated by long sojourns in desensitized states (Sakmann et al., 1980; Colquhoun and Ogden, 1988) (for glycine receptors, see Takahashi and Momiyama, 1991; Beato et al., 2004). Because each patch contained relatively few clusters, the distributions in Figure 1 contain few of these (desensitized) intercluster shut times. These intervals were not fitted but are clearly distinct from the much shorter intracluster shuttings. Hence, gaps were classed as between clusters if longer than 1000 $\mathrm{ms}$ at $30 \mu \mathrm{M}, 60 \mathrm{~ms}$ at $100 \mu \mathrm{M}$, and $20 \mathrm{~ms}$ at $1000 \mu \mathrm{M}$.

At all concentrations, most of the shuttings belong to the shortest component, which has a time constant (at $10 \mu \mathrm{M}$ ) of approximately $14 \mu$ s (Table 1 ). This is much shorter than our experimental resolution of $30 \mu \mathrm{s}$, and many (approximately $88 \%$ ) such gaps would be missed. Nevertheless, given that $67 \%$ of shut times are "in the fast component," in a typical run with 15,000 transitions the number of " $14 \mu$ s component shuttings" that are above $30 \mu \mathrm{s}$ is almost 700, which would predict a coefficient of variation of only $4 \%$ for estimation of the $14 \mu$ s time constant. Therefore, the reproducibility of the estimates of the fastest time constant, shown in Table 1, is not surprising.

The distribution of apparent open times (Fig. 1) shows a shift toward longer apparent open times at the higher concentrations. This results from a decline in the relative area of the faster component (Table 1), which can no longer be resolved at $1000 \mu \mathrm{M}$. Time constants of both components were essentially unchanged with concentration, suggesting that dissociation from the open states is relatively slow. As in homomeric channels, the faster component may therefore correspond to partially liganded open states of the receptor (Beato et al., 2002).

\section{The $P_{\text {open }}$ curve}

Figure 2 illustrates how the glycine concentration- $P_{\text {open }}$ curve was obtained from the analysis of clusters of openings evoked by 30-1000 $\mu \mathrm{M}$ glycine. The expanded traces on the right-hand side also show the consistency of the single-channel conductance and the lack of conductance sublevels in $\alpha 1 \beta$ heteromeric receptors, in accord with Bormann et al. (1993). In all patches, the distribution of fitted amplitudes for single-channel currents could be fitted well with a single Gaussian density (3.1 $\pm 0.1 \mathrm{pA}$; data not shown).

Experimental $P_{\text {open }}$ values are plotted against glycine concentration in Figure $2 F$, where they are fitted by a Hill equation. This plot makes no postulates about mechanism, so the exact correction for missed events that is used for subsequent $P_{\text {open }}$ plots 
Table 1. Dwell-time distributions

\begin{tabular}{|c|c|c|c|c|c|c|}
\hline \multicolumn{7}{|c|}{ Apparent open periods } \\
\hline $\operatorname{Gly}(\mu \mathrm{m})[n]$ & $\tau_{1}(\mathrm{~ms})$ [area (\%)] & $\tau_{2}(\mathrm{~ms})$ [area (\%)] & & & & \\
\hline $\begin{array}{l}10[4] \\
30[4] \\
100[4] \\
1000[4]\end{array}$ & $\begin{array}{l}0.69 \pm 0.06[60 \pm 3] \\
0.58 \pm 0.09[52 \pm 3] \\
0.42 \pm 0.03[16 \pm 1]\end{array}$ & $\begin{array}{l}2.7 \pm 0.1[40 \pm 3] \\
2.6 \pm 0.2[48 \pm 3] \\
3.1 \pm 0.4[84 \pm 1] \\
6.1 \pm 0.2[100]\end{array}$ & & & & \\
\hline \multicolumn{7}{|c|}{ Apparent shut times } \\
\hline Gly $(\mu \mathrm{m})[n]$ & $\tau_{1}(\mathrm{~ms})[\operatorname{area}(\%)]$ & $\tau_{2}(\mathrm{~ms})[\operatorname{area}(\%)]$ & $\tau_{3}(\mathrm{~ms})$ [area (\%)] & $\tau_{4}(\mathrm{~ms})[\operatorname{area}(\%)]$ & $\tau_{5}(\mathrm{~ms})[\operatorname{area}(\%)]$ & $\tau_{6}(\mathrm{~ms})[\operatorname{area}(\%)]$ \\
\hline $\begin{array}{l}10[4] \\
30[4] \\
100[4] \\
1000[4]\end{array}$ & $\begin{array}{l}0.014 \pm 0.001[67 \pm 4] \\
0.015 \pm 0.001[68 \pm 4] \\
0.014 \pm 0.001[86 \pm 2] \\
0.012 \pm 0.001[94 \pm 1]\end{array}$ & $\begin{array}{r}0.10 \pm 0.01[11 \pm 2] \\
0.12 \pm 0.01[17 \pm 2] \\
0.10 \pm 0.01[11 \pm 2] \\
0.084 \pm 0.004[5 \pm 1]\end{array}$ & $\begin{array}{l}0.28 \pm 0.02[11 \pm 1] \\
0.35 \pm 0.05[8.3 \pm 1.6] \\
0.50 \pm 0.04[2.4 \pm 0.3]\end{array}$ & $\begin{aligned} 1.5 & \pm 0.2[1.2 \pm 0.3] \\
4 & \pm 1[1.0 \pm 0.3] \\
6 & \pm 1[1.1 \pm 0.2] \\
1.7 & \pm 0.2[0.35 \pm 0.03]\end{aligned}$ & $\begin{array}{l}130 \pm 20[4.9 \pm 0.4] \\
120 \pm 20[6.1 \pm 0.9]\end{array}$ & $380 \pm 40[3.9 \pm 0.6]$ \\
\hline
\end{tabular}

Distributions were fitted with mixtures of exponential probability density functions. The number of experiments at each concentration is indicated in brackets in the first column. The time constant of each component and its relative area (in brackets) are given as mean \pm SD of the mean.

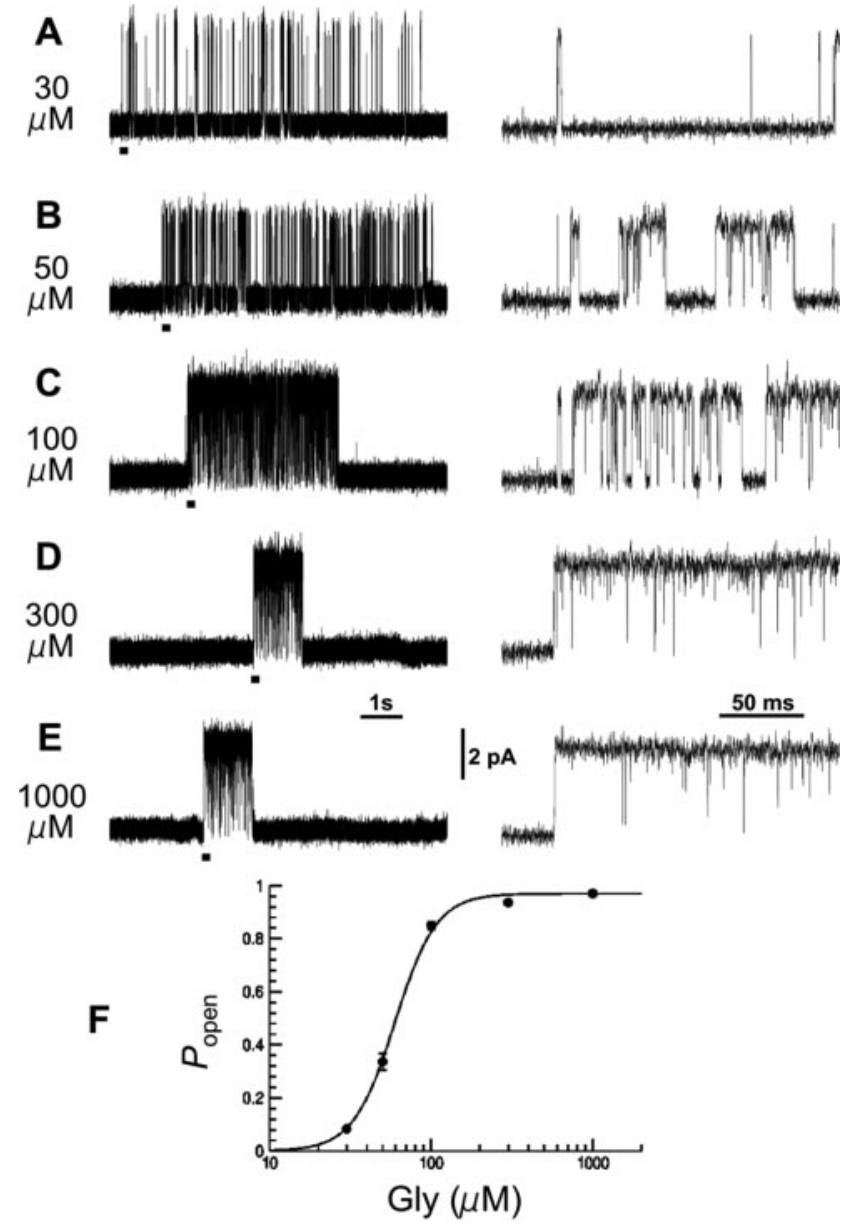

Figure 2. Concentration dependence of the open probability of clusters of $\alpha 1 \beta$ channel openings. $A-E$, Representative clusters elicited by $30,50,100,300$, and $1000 \mu \mathrm{m}$ glycine. The bars under the traces on the left indicate the regions shown on the right on an expanded time scale. $F$, The $P_{\text {open }}$ concentration-response curve to glycine. The curve shows an empirical fit of the Hill equation (weighted least squares). The observed points in this plot (unlike later plots) have been corrected approximately for missed short shuttings (see Results). $P_{\text {open }}$ values are obtained for each patch from the ratio between the total open time (obtained from records idealized by time-course fitting) and the total duration of the clusters. The points are averages of three to four patches (17-91 clusters) (Table 2) for each glycine concentration.

cannot be used. Therefore, for this plot only, values of $P_{\text {open }}$ were corrected for missed shut times by estimating the approximate time occupied by the missed shut times, by extrapolation to $t=0$ of the shut time distribution (Colquhoun and Sakmann, 1985).
This gave a maximum $P_{\text {open }}$ of 0.97 (two-unit likelihood interval, $0.968-0.971$ ), an $E C_{50}$ of $60 \mu \mathrm{M}$ (two-unit likelihood interval, 57-62 $\mu \mathrm{M}$ ), and a Hill slope of 3.4 (two-unit likelihood interval from residuals, 3.1-3.7); for normally distributed variables, two-unit likelihood intervals are the same as $95 \%$ confidence intervals (Colquhoun and Sigworth, 1995). The Hill equation is not, of course, the correct equation to fit (it describes no possible physical mechanism), so the slope that it gives is in no sense the "right" value; it was fitted merely to obtain an empirical estimate of the steepness of the $P_{\text {open }}$ curve for comparison with the predictions of postulated mechanisms (this consideration affects also the calculation of confidence intervals). In fact a Hill slope greater than 3 is not possible for any of the mechanisms that we discuss here. Thus there is some uncertainty in our Hill slope estimate, but what actually matters is not so much the value for a Hill slope but rather how well the whole predicted $P_{\text {open }}$ curve fits the observed points in Figures $4-8$ and 10. The predictions of Hill slope from fits (Figs. 4-8 and 12) are based on apparent (HJC) values, and therefore, strictly speaking, they are not exactly comparable with the value found from Figure 2, but the difference is very small (Table 2 ).

Although the observed $E C_{50}$ value, $60 \mu \mathrm{M}$, is well within the range reported for $\alpha 1 \beta$ heteromers in HEK293 cells (between 18 and $74 \mu \mathrm{M}$ ) (Bormann et al., 1993; Handford et al., 1996; Rees et al., 2002), the Hill slope is at the top end of the range of values in the literature (1.8-3.0) (Handford et al., 1996; Legendre, 1998; Shan et al., 2001). Most previous values were obtained by macroscopic methods and are inevitably more affected by desensitization than a $P_{\text {open }}$ concentration-response curve. Thus, discrepancies between the two methods are not surprising. Nevertheless, because of the importance of the $P_{\text {open }}$ curve as a criterion for adequacy of a postulated mechanism, we investigated the potential sources of bias in our measurements.

First, $P_{\text {open }}$ values may be overestimated if the group of openings classified as a cluster originates from more than one channel, i.e., it really is two interlaced clusters. Unless the open probability of these two clusters is very low, their superposition would give rise to double openings, which would make us discard the resulting cluster. The lowest $P_{\text {open }}$ that we measured was 0.084 (at 30 $\mu \mathrm{M}$ glycine). If clusters of this overall $P_{\text {open }}$ were produced by two active channels, the mean number of openings in a run of single openings would be only 23 , with a probability of approximately 0.01 of observing a run containing more than 104 single openings (Colquhoun and Hawkes, 1990). Most clusters (e.g., 22 of 27 at 30 $\mu \mathrm{M})$ contained far more openings than this without a double opening being seen. 
Table 2. Properties of clusters of $\alpha 1 \beta$ channel openings at different glycine concentrations

\begin{tabular}{llllll}
\hline $\begin{array}{l}\text { Gly } \\
(\mu \mathrm{m})\end{array}$ & $\begin{array}{l}\text { Number of } \\
\text { clusters }\end{array}$ & $\begin{array}{l}\text { Number of } \\
\text { patches }\end{array}$ & $\begin{array}{l}\text { Cluster length } \\
(\mathrm{s})\end{array}$ & $\begin{array}{l}P_{\text {open }} \\
\text { (uncorrected) }\end{array}$ & $\begin{array}{l}P_{\text {open }} \\
\text { (corrected for missed events) }\end{array}$ \\
\hline 30 & 27 & 4 & $14 \pm 2$ & $0.084 \pm 0.007$ & $0.083 \pm 0.007$ \\
50 & 17 & 3 & $4.5 \pm 0.9$ & $0.340 \pm 0.031$ & $0.336 \pm 0.03$ \\
100 & 38 & 4 & $1.6 \pm 0.3$ & $0.862 \pm 0.013$ & $0.850 \pm 0.014$ \\
300 & 63 & 3 & $0.8 \pm 0.1$ & $0.946 \pm 0.006$ & $0.935 \pm 0.004$ \\
1000 & 91 & 4 & $1.0 \pm 0.1$ & $0.983 \pm 0.002$ & $0.9706 \pm 0.0007$ \\
\hline
\end{tabular}

$P_{\text {open }}$ values are obtained for each patch as ratios of total open time and total cluster duration (calculated from recordings idealized by time-course fitting). Values are means $\pm S D$ of the mean. Hill equation fits of the apparent $P_{\text {open }}$ values (i.e., uncorrected) gave a Hill slope of 3.5 (2-unit likelihood interval: 3.3-3.8), a maximum $P_{\text {open }}$ of 0.98 (2-unit likelihood interval, $\left.0.976-0.984\right)$, and an $E C_{50}$ of $59 \mu \mathrm{m}$ (2-unit likelihood interval, $56-61 \mu \mathrm{m})$.

\section{Scheme 1}

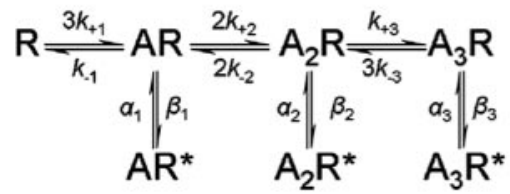

Scheme 2

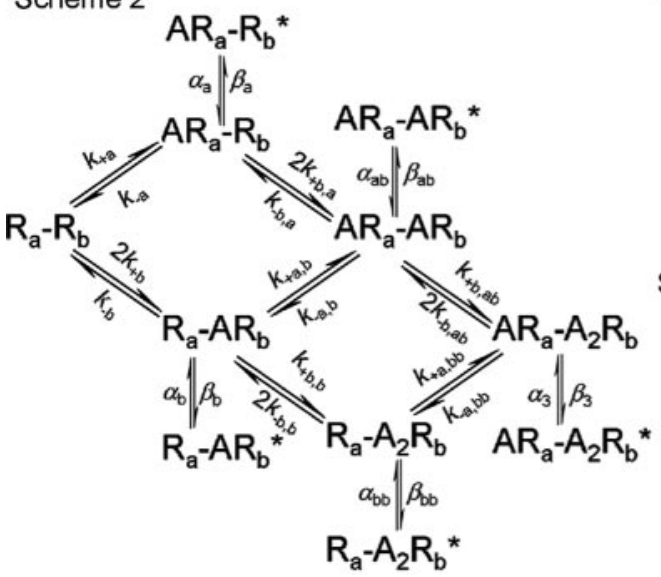

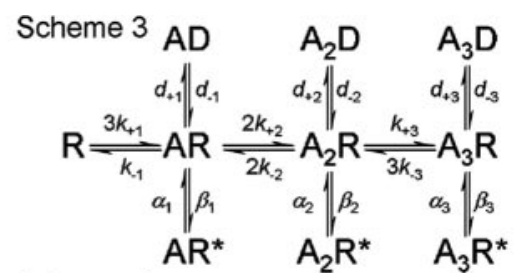

Scheme 4

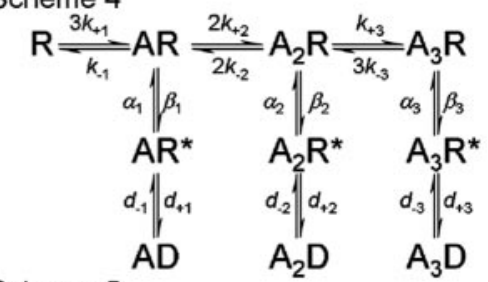

Scheme 5

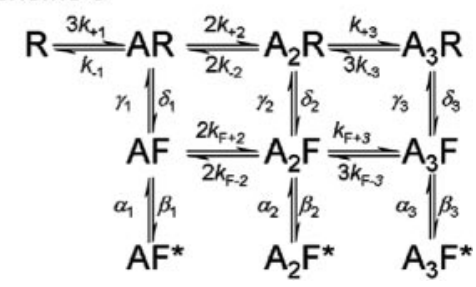

Figure 3. Some of the kinetic schemes tested for $\alpha 1 \beta$ glycine channels. Agonist molecules are indicated by $A$, resting states of the receptor by $R$, and open states by an asterisk. Note that in scheme 2 , the receptor is represented by $R_{a}-R_{b}$ to indicate that it contains two different types of binding sites ( $a$ and $b$ ). In schemes $3-5$, shut states of the receptor are denoted $R, D$ (desensitized), and $F$ (flipped, i.e., in the pre-opening conformation; see Results). Open channels are indicated by an asterisk ( $\mathrm{R}^{*}$ or $\mathrm{F}^{*}$ ). The names of the rate constants for the different steps of the reactions are shown, and the statistical factors in the binding rate constants have been included. These are needed to get the actual transition rates in the $\mathbf{Q}$ matrix, taking into account the number of sites available for each binding step.

Another way in which $P_{\text {open }}$ could be overestimated would be to choose inappropriately low $t_{\text {crit }}$ values, because this would lead us to divide one cluster into several. To test for errors in the $t_{\text {crit }}$ calculation, we tried increasing or decreasing its value by a factor of 5, but found that the $P_{\text {open }}$ values barely changed (by $3 \%$, on average). Finally, the bias caused by missing short shut times was corrected by an approximate extrapolation method in Figure 2, but for all of the fits, the apparent (HJC) $P_{\text {open }}$ value, with exact allowance for missed events, was calculated from Equations 2-4.

\section{Fits of putative mechanisms}

The number of binding sites

Heteromeric glycine receptors contain three copies of the $\alpha$ subunit and two copies of the $\beta$ subunit (Kuhse et al., 1993; Burzomato et al., 2003). Given that the $\alpha$ subunit appears to be essential to the formation of the binding site (Laube et al., 2002), this stoichiometry implies a maximum number of three binding sites.
First we examined the possibility that the receptor contains two agonist binding sites, as suggested by Legendre (1998) on the basis of the shape of the rise time of macroscopic currents evoked by glycine concentration jumps on zebrafish native receptors. We tested several schemes with two binding steps and a minimum of two open states and investigated all possible combinations of connectivity and interaction between binding sites. Mechanisms tested thus included the possibility that the two binding sites are identical to start with [a "sequential" scheme, similar to scheme 1, which has three binding sites (Fig. 3)] or are different. In both schemes we also incorporated the possibility of interaction between binding sites (i.e., cooperativity in binding). Another variant tested was the inclusion of an additional fully bound reluctant shut state leading into an additional open state (Legendre, 1998). None of these variations produced a good description of our data. The predicted Hill slopes, inevitably all less than 2, were much shallower than the value that we observed. In terms of the maximum likelihood achieved, all three different mechanisms with only two binding sites were ranked in the lower $50 \%$ of all mechanisms that we tested.

Therefore, we proceeded to test mechanisms with three binding sites: these are shown in Figure 3, together with the names of the rate constants. The appropriate "statistical factors" are shown with the rate constants in Figure 3. These factors take into account the number of sites available for each (un)binding step, so that $k_{+\mathrm{n}}$ and $k_{-\mathrm{n}}$ are the association and dissociation rate constants per site for the $n$th binding. For example, if three identical sites are occupied, any one of the three bound molecules may dissociate, so the actual transition rate is $3 k_{-3} s^{-1}$. To maintain generality, all mechanisms tested include an open state for each ligation state of the receptor.

The simplest mechanism is scheme 1 (Fig. 3). This has sequential binding to three glycine sites that are identical to start with. In the simplest case, the binding sites do not interact with each other; i.e., the binding and unbinding rate constants for a site are not affected by whether the other sites are occupied. The assumption of independent sites is implemented by applying the following constraints to the values of the binding rate constants during the fitting:

$$
k_{+1}=k_{+2}=k_{+3}, \quad k_{-1}=k_{-2}=k_{-3} .
$$

The results of fitting this model are shown in Figure 4 (the constraint of independent sites is denoted by the three identical equilibrium constants $K$ in the scheme in $A$ ). 
For each mechanism, the HJCFIT program (see Materials and Methods) obtained optimized rate constant values by maximizing the likelihood of the whole sequence of openings and shuttings in a recording. The data input consisted of sets of recordings (idealized by time course fitting), one at each of four different agonist concentrations (10, 30, 100, and $1000 \mu \mathrm{M})$, together with the value of the experimental resolution, to allow full missed-event correction. The output consists of a set of maximum likelihood estimates of rate constants. These values are then used to judge the adequacy of the mechanism (see Materials and Methods). Figure $4 B$, top two rows, shows apparent open-time and apparent shuttime distributions (same patches as Fig. 1) together with the predicted (HJC) distributions (continuous lines) superimposed on the data (the predicted distribution at perfect resolution is also plotted, as a dashed line; see Materials and Methods). The failure of this mechanism to describe the experimental observations is very clear, particularly for shut times. Note that the data in these histograms are the same as in Figure 1, where the fits shown are empirical (mixtures of exponentials fitted to each distribution separately), rather than global mechanism fits using the whole sequence of events (and hence the full information contained in the data) for four concentrations simultaneously.

The plot of mean open time, conditional on adjacent shut time (see Materials and Methods), is shown in Figure $4 B$, bottom row (see Materials and Methods for details). The conditional mean open times predicted for this mechanism (open circles) are compared with the experimental points (filled circles joined by continuous straight lines). Again, scheme 1 with identical and noninteracting binding sites does not describe adequately this aspect of the data, because the predicted values lie well below the experimental points at $1000 \mu \mathrm{M}$ glycine. The final criterion is provided by the apparent $P_{\text {open }}$-concentration curve (Fig. $4 C$ ), which is a poor fit, being clearly too shallow (a Hill slope of 1.84 is predicted at the $E C_{50}$ ).

The nature of the binding sites

The mechanism tested above (scheme 1, with no interaction between the binding sites) is clearly unsatisfactory. Two possible reasons for this inadequacy are that the binding sites might not be identical before glycine binds and/or that they may interact. We next tested these two hypotheses.

In ligand-gated ion channels in the nicotinic superfamily, such as glycine channels, agonist binding sites are believed to be at the interface between an $\alpha$ subunit and the adjacent subunit in the counterclockwise direction (Brejc et al., 2001). In a glycine heteromeric receptor, it is generally assumed that the three $\alpha$ and two $\beta$ subunits are arranged around the pore in a $\alpha \beta \alpha \beta \alpha$ topology. This implies that of the three binding sites, two are formed by an $\alpha \beta$ interface and one by an $\alpha \alpha$ interface. In addition, the two
B
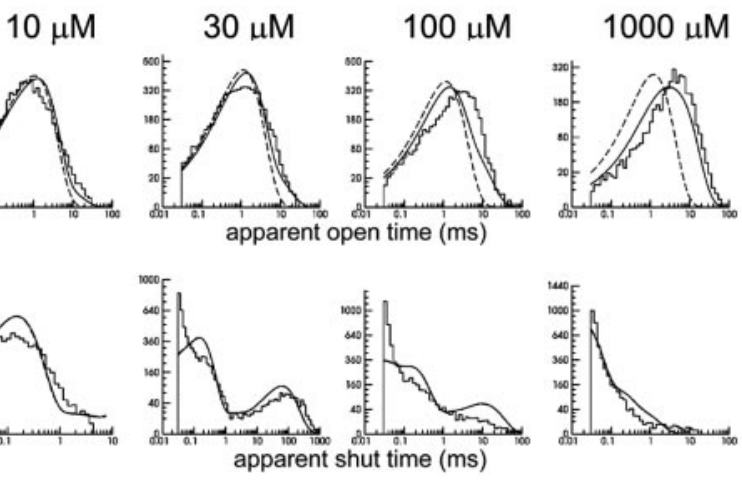

으
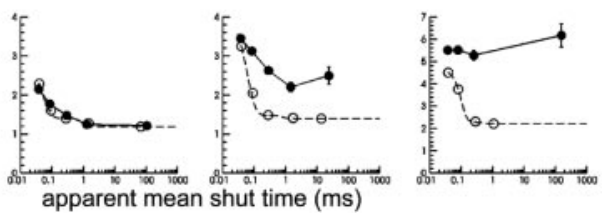

Figure 4. Fitting of a scheme with three identical binding sites (scheme 1) to $\alpha 1 \beta$ glycine channel activations (8 free parameters). $A$, Scheme 1 was fitted with constraints (Eq. 5) that ensured that the association and dissociation rate constants were the dhe binding steps. $B$, Comparison of the predictions of the fit with the experimental data. The mechanism was fitted distributions (note that only shut times below $t_{\text {crit }}$ are fitted; see Materials and Methods). The solid lines are predicted (HJC) distributions calculated from the mechanism, the resolution, and the values of the rate constants that were found to maximize the dhese plots illustrate the correlation between the duration of adjacent open and 作 - concentration curve predicted by the fitted scheme and rate constants taking into account the effect of missed events (from Eqs. 2-4). The dashed line is the ideal curve expected if no events were missed. In this case, the predictions of the fitted mechanism and rates are quite poor, so this scheme is unlikely to be the real activation mechanism of the channel.

$\alpha$ subunits that participate in the $\alpha \beta$ sites are nonequivalent in principle, because one contributes also to the $\alpha \alpha$ site and the other does not. Thus one of the $\alpha \beta$ sites is proximal (to the $\alpha \alpha$ site) and one is distal.

The first test of the hypothesis that the three binding sites are initially different was performed by fitting scheme 2 (Fig. 3 ). This mechanism incorporates two types of binding sites (one $\alpha \alpha$ and two identical $\alpha \beta$ sites) and has an open state connected to each ligation state of the receptor, but we retained the hypothesis of no interaction between the sites. Thus binding and unbinding rate constants for the $\alpha \alpha$ site were constrained to keep the same value, regardless of whether the $\alpha \beta$ sites are occupied; similar constraints were imposed for the $\alpha \beta$ sites, thus:

$$
\begin{gathered}
k_{+\mathrm{a}}=k_{+\mathrm{a}, \mathrm{b}}=k_{+\mathrm{a}, \mathrm{bb}}, \quad k_{-\mathrm{a}}=k_{-\mathrm{a}, \mathrm{b}}=k_{-\mathrm{a}, \mathrm{bb}}, \\
k_{+\mathrm{b}}=k_{+\mathrm{b}, \mathrm{a}}=k_{+\mathrm{b}, \mathrm{b}}=k_{+\mathrm{b}, \mathrm{ab}}, \\
k_{-\mathrm{b}}=k_{-\mathrm{b}, \mathrm{a}}=k_{-\mathrm{b}, \mathrm{b}}=k_{-\mathrm{b}, \mathrm{ab}} .
\end{gathered}
$$

Note that these constraints imply that the principle of microscopic reversibility is respected for all cycles in the scheme (Colquhoun et al., 2004) and leave 14 rate constants to be fitted as free parameters. The results of this fit are illustrated in Figure 5 (same set of experiments as shown in Figs. 1 and 4). The mecha- 


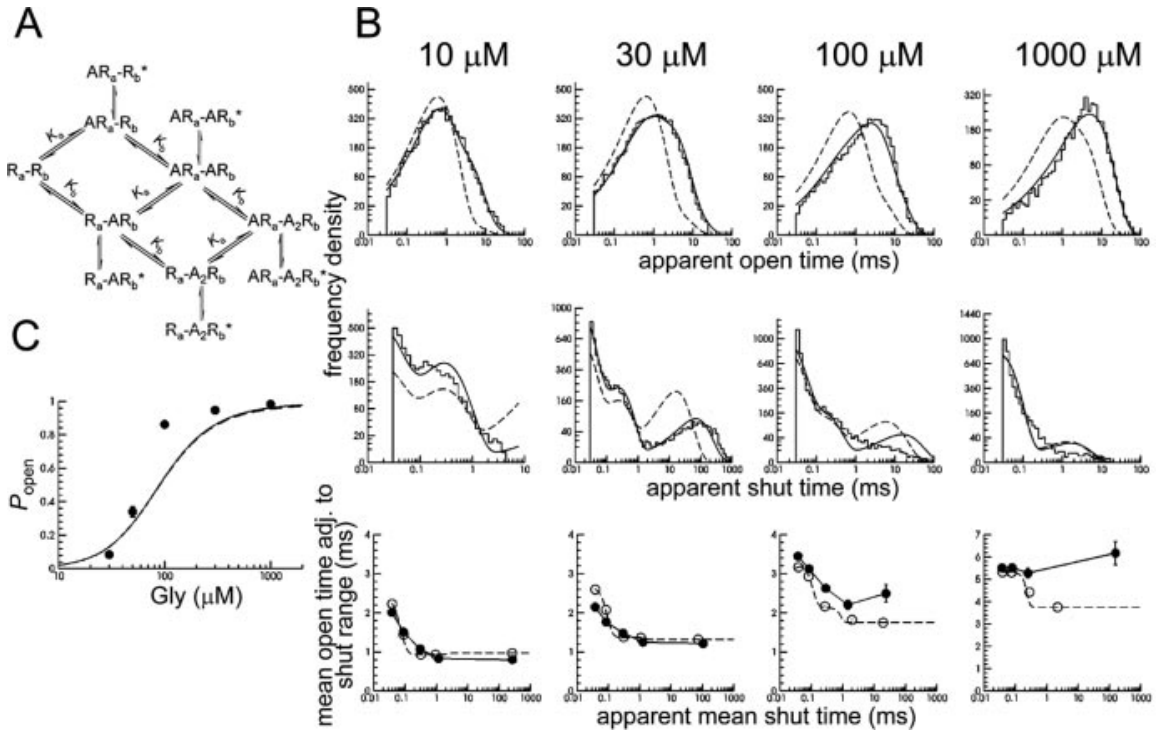

Figure 5. Test of the possibility that agonist binding sites are initially different: a scheme with two types of binding sites (scheme 2, 14 free parameters; constraints as in Eq. 6). A, Scheme 2 incorporates two types of binding sites, termed a and b, corresponding to the $\alpha \alpha$ site and the two $\alpha \beta$ sites, respectively. Binding rate constants at each site are postulated not to be affected by occupancy of other sites, and therefore the $\alpha \alpha$ site has a constant affinity of $1 / K_{\mathrm{a}}$ and each $\alpha \beta$ site a constant affinity of $1 / K_{\mathrm{b}} . B$, Comparison of observed and predicted dwell-time distributions and open-shut time correlations, as in Figure 4. $C$, $P_{\text {open }}$ - concentration data and the concentration-response curve predicted from the mechanism fit, as in Figure 4. The obvious discrepancies between experimental observations and model predictions in $B$ (especially at the higher concentrations) and $C$ indicate that scheme 2 fails to describe the data.

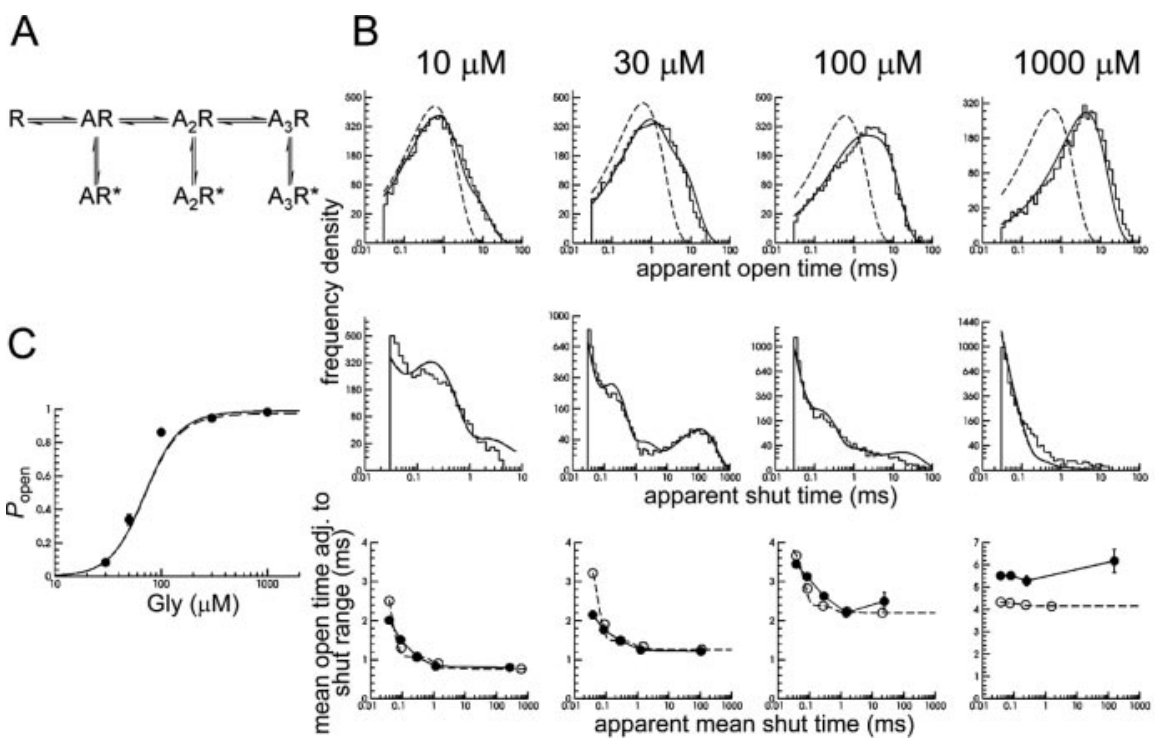

Figure 6. Test of a mechanism in which the affinity of the binding sites appears to be affected by the level of occupancy: a scheme with three binding sites and no constrained parameters (12 free parameters). A, All rate constants in scheme 1 were estimated as free parameters. $B$, Comparison of observed and predicted dwell-time distributions and open-shut time correlations, as in Figure 4. $C, P_{\text {open }}$ - concentration data and the concentration-response curve predicted from the mechanism fit. Comparing this fit with that of Figure 4 shows an improvement that is consistent across all forms of data display. Nevertheless, the fit is still inadequate for data at $1000 \mu \mathrm{m}$ and for the fast component of the shut-time distribution at $10 \mu \mathrm{m}$.
We next examined a scheme in which all three binding sites were different initially ( $\alpha \alpha, \alpha \beta$ proximal, $\alpha \beta$ distal) but were not allowed to interact with each other. Despite the large number of states and free parameters (16 and 20, respectively), this scheme again proved inadequate, with problems similar to those found for scheme 2 (i.e., poor $P_{\text {open }}$ curve with a low predicted Hill slope and a poor description of the shut-time distributions; data not shown). Thus we concluded that if there is any difference between sites in the resting state, it is too small to be detected.

Interaction between the binding sites

Most of the mechanisms tested so far have not included the possibility that the agonist binding sites may interact, namely that the binding rate constants for one site may be affected by whether the other sites are occupied, before the channel is open. To test this hypothesis in the simplest form, we fitted again scheme 1, this time relieving all constraints and therefore fitting all of the 12 rate constants as free parameters. A comparison of the resulting fit (Fig. 6) with the fit of the same scheme with the constraint of noninteraction (Fig. 4) shows a considerable improvement in the description of single-channel behavior. The rate constant values obtained in the fit (Table 3 ) show that binding affinity increases monotonically with each binding step by approximately fivefold. Gating efficacy also increases as more binding sites are occupied. Most importantly, this is the first mechanism fit that predicts a Hill slope $\left(2.63\right.$ at the $\left.E C_{50}\right)$ that approaches the observed one (Fig. $6 C$ ). Note also that all rate constant estimates were well defined (Table 3 ), unlike the rates found previously for the fit of scheme 2, which has exactly the same number of free parameters.

This suggests, as in our recent report for glycine $\alpha 1$ homomeric channels (Beato et al., 2004), that interaction between the agonist binding sites is an essential feature in the activation of heteromeric $\alpha 1 \beta$ glycine receptors. Furthermore, good fits can be obtained without having to postulate that the binding sites are nonequivalent in the resting state. nism in Figure $5 A$ fails to describe the observed single-channel behavior. This is particularly obvious at high agonist concentrations, where predictions for apparent shut-time distributions and conditional mean open times are poor. In addition, the predicted $P_{\text {open }}$ curve is a poor fit, with a Hill slope that is still too low (1.69). We found also that rate constant estimates for this scheme were very variable from one experimental set to the other (data not shown).

\section{Additional bound shut states}

Although releasing the constraint of independence of the binding sites improved the quality of the fit by scheme 1, as shown clearly in Figure $6 B$, this mechanism is still inadequate in describing shut-time distributions and high concentration open-shut correlations.

In an effort to get better fits, we next added additional bound 
Table 3. Estimated parameters from the fit of scheme 1 (with no constraints) to single-channel data

\begin{tabular}{|c|c|c|c|}
\hline & Units & Mean estimates & CV (\%) \\
\hline$\alpha_{1}$ & $s^{-1}$ & 3700 & 12 \\
\hline$\beta_{1}$ & $s^{-1}$ & 32 & 11 \\
\hline$\alpha_{2}$ & $s^{-1}$ & 1310 & 4 \\
\hline$\beta_{2}$ & $s^{-1}$ & 4600 & 8 \\
\hline$\alpha_{3}$ & $s^{-1}$ & 1900 & 11 \\
\hline$\beta_{3}$ & $s^{-1}$ & 80000 & 7 \\
\hline$k_{-1}$ & $s^{-1}$ & 490 & 6 \\
\hline$k_{+1}$ & $M^{-1} S^{-1}$ & $0.38 \times 10^{6}$ & 13 \\
\hline$k_{-2}$ & $s^{-1}$ & 750 & 6 \\
\hline$k_{+2}$ & $M^{-1} S^{-1}$ & $3.3 \times 10^{6}$ & 3 \\
\hline$k_{-3}$ & $s^{-1}$ & 1800 & 6 \\
\hline$k_{+3}$ & $M^{-1} S^{-1}$ & $37 \times 10^{6}$ & 14 \\
\hline$E_{1}$ & & $8.5 \times 10^{-3}$ & 2 \\
\hline$E_{2}$ & & 3.5 & 12 \\
\hline$E_{3}$ & & 42 & 3 \\
\hline$K_{1}$ & $\mu \mathrm{M}$ & 1300 & 17 \\
\hline$K_{2}$ & $\mu \mathrm{M}$ & 230 & 4 \\
\hline$K_{3}$ & $\mu \mathrm{M}$ & 48 & 10 \\
\hline$E C_{50}$ & $\mu \mathrm{M}$ & 70 & 3 \\
\hline$n_{\mathrm{H}}$ & & 2.63 & 2 \\
\hline
\end{tabular}

The values given for the rate constants are averages of three estimates, each from the fit to a set of four experiments at different glycine concentrations. All 12 rate constants (Fig. 3) were fitted freely in HJCFIT. Values for the dissociation constants $\left(K_{n}=k_{-n} / k_{+}\right)$and the efficacy at different levels of ligation $\left(E_{n}=\beta_{n} / \alpha_{n}\right)$ as well as $E C_{50}$ and the Hill slope were calculated from the rate constants. Equilibrium constants were calculated separately for each set and then averaged (so the mean values for equilibrium constants are not identical to the ratio of the mean rate constants in this and subsequent tables). The prediction of the data, shown in Figure 6 for one data set, was inadequate, although much better than when the binding was constrained to be independent $\left(K_{1}=K_{2}=K_{3}\right)$ (Fig. 4).

shut states, the lifetimes of which are not concentration dependent. In one form or another, such shut states (often referred to as "short-lived desensitized" states) have been found to be an essential feature of activation mechanisms for several receptors in this superfamily. The simplest form, used for the description of single-channel behavior for the muscle nicotinic receptor (Salamone et al., 1999; Hatton et al., 2003), involves one distal shut state (connected to the fully liganded open or shut state). The more general form in this family of mechanisms is that developed by Jones and Westbrook (1995) to explain the macroscopic behavior of $\mathrm{GABA}_{\mathrm{A}}$ receptors in response to paired short pulses of agonists and incorporates one extra shut state at each level of ligation. Although formally similar to the Jones-Westbrook mechanism, in that extra shut states appear within an individual channel activation, the shut states denoted " $D$ " in scheme 3 are more short-lived than those for $\mathrm{GABA}_{\mathrm{A}}$ and do not account for slow components of macroscopic desensitization.

We therefore proceeded to test whether adding extra shut states to the unconstrained form of scheme 1 helped account for observed channel behavior. Only a slight improvement was detected when a single shut state was added in a position distal to either $A_{3} R$ or $A_{3} R^{*}$ (Salamone et al., 1999; Hatton et al., 2003) (data not shown). Adding a distal shut state to scheme 1 or scheme 2 (while keeping the constraint of binding site independence) also failed to produce any detectable improvements in the quality of the fit.

The results of the global fit of our data with the more general model (Jones and Westbrook, 1995) adapted to three binding sites (Fig. 3, scheme 3) are shown in Figure 7 and Table 4. Comparison of the plots in Figure 7, $B$ and $C$, with those in Figure 6 clearly indicates that scheme 3 is much better at describing the behavior of $\alpha 1 \beta$ channels than scheme 1 without constraints. A substantial improvement is visible in the accuracy with which HJC distributions predict shut-time distributions and the condi- tional mean plot at $1000 \mu \mathrm{M}$ glycine. The quality of the description of the dependence of the apparent cluster $P_{\text {open }}$ on glycine concentration is similar for schemes 1 and 3 . It must be noted that this improvement is achieved at the price of an increase in the number of free parameters in the mechanism (from 12 to 18).

\section{Other mechanisms with three extra shut states}

The success of the mechanism in scheme 3 in accounting for the single-channel properties of heteromeric glycine channels led us to consider related schemes, all with three binding steps, three open states, and three extra shut states. These schemes differ only in how the states are connected to each other, and the two possible alternative positions for the open states are shown in Figure 3 as schemes 4 and 5 . In the first instance, we fitted both scheme 4 and a reduced scheme 5 (this means that no binding or unbinding was allowed between the F states in scheme 5) with no constraints. The results of these fits were good, nearly as good as those of fitting scheme 3 with no constraints, because both models described the data well and predicted a Hill slope value of 2.532.69 (data not shown). Note that all three of these mechanisms have the same number of free parameters (18). Binding site interaction was implied by all three schemes, because constraining the binding sites to be independent greatly reduced the quality of the fits (for example, predicting shallow Hill slopes of 1.8-1.9, 14 free parameters; data not shown).

Schemes 3, 4, and 5 with one open state omitted were also tested, on the grounds that the empirical fit of apparent open times with two exponentials (Fig. 1, Table 1) suggested that it might not be necessary to postulate three open states. The fits of apparent open times with two open states were visibly worse than with three open states; these models are nested, and likelihood ratio tests showed that two open states were unacceptable. The empirical fit of exponentials does not resolve all of the open time components, possibly because open times below approximately 3 $t_{\text {res }}(90 \mu \mathrm{s}$ in this case) are not described, in principle, by a mixture of exponential distributions when many short events are missed (Hawkes et al., 1990, 1992). This might also explain why the fastest component of shut times that was fitted empirically was approximately $14 \mu \mathrm{s}$, whereas the fits in Figures 7 and 8 suggest that it should be nearer $8 \mu$ s.

\section{A conformation change produced by binding while the channel is still shut: the flip mechanism}

So far, we found that the data consistently require binding sites to interact, such that the affinity for binding to the shut state appears to increase as more molecules are bound. The monotonic increase in affinity with increasing ligation $\left(K_{1}>K_{2}>K_{3}\right)$ found for the unconstrained fit of scheme 1 (Table 3 ) suggests that the appearance of interaction may occur secondary to a concerted (all subunit) conformation change, in the way first postulated by Wyman and Allen (1951) and subsequently applied to enzymes (Monod et al., 1965). Wyman and Allen (1951) suggested that if a protein existed in two conformations, one of which has a higher affinity for a ligand than the other, then the ligand would cause a shift to the higher affinity form. This would give the appearance of increasing overall affinity as more ligand was bound, even if all of the sites on each conformation were independent (and so had the same affinity for the ligand, regardless of the number of the other sites that were already occupied).

This argument leads naturally to scheme 5 , which we refer to, for brevity, as the flip mechanism (Figs. 3, scheme 5, 12A). This mechanism postulates that agonist binding can favor a conformation change that occurs before, and separately from, the chan- 
A

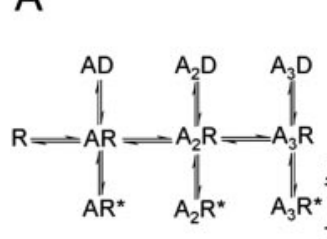

B $10 \mu \mathrm{M}$
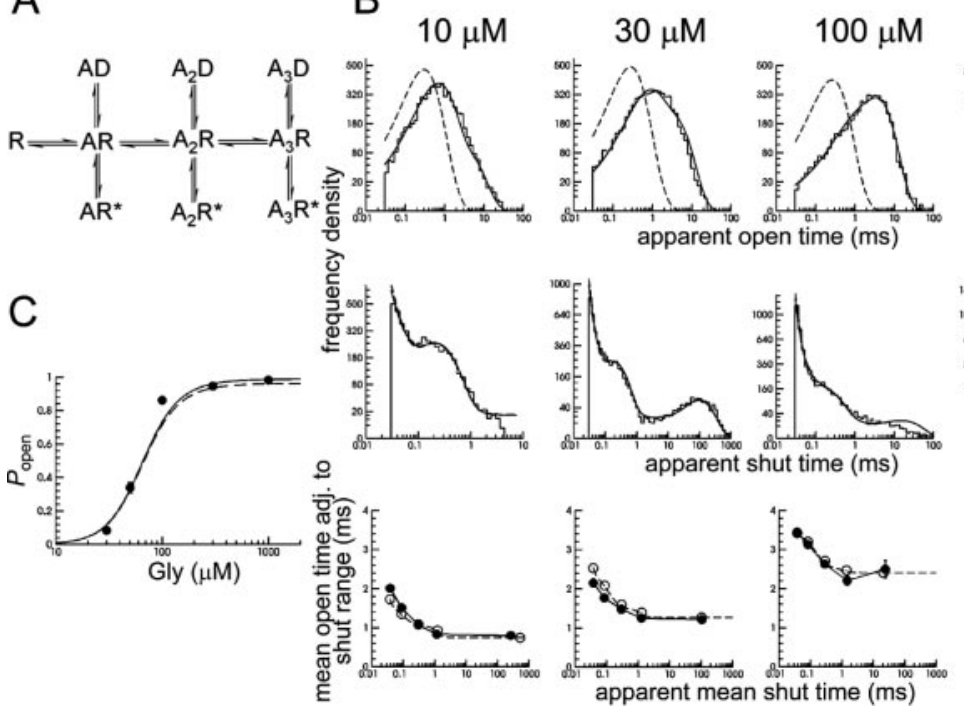

Figure 7. Test of a mechanism with three additional shut states and no constrained parameters (18 free parameters). $A$, Scheme 3 , showing the additional shut states as D. Access to D states is not concentration dependent. $B$ and $C$ are as in Figure 4 . Note that the predictions of this model are much closer to the observed dwell-time distributions and open-shut correlations than those of scheme 1 with no constraints (Fig. 6).

Table 4. Estimated parameters from the fit of scheme 3 to single-channel data

\begin{tabular}{|c|c|c|c|}
\hline & Unit & Mean estimates & CV (\%) \\
\hline$\alpha_{1}$ & $s^{-1}$ & 3400 & 11 \\
\hline$\beta_{1}$ & $s^{-1}$ & 400 & 45 \\
\hline$\alpha_{2}$ & $s^{-1}$ & 2200 & 11 \\
\hline$\beta_{2}$ & $s^{-1}$ & 28000 & 10 \\
\hline$\alpha_{3}$ & $s^{-1}$ & 3700 & 9 \\
\hline$\beta_{3}$ & $s^{-1}$ & 112000 & 3 \\
\hline$d_{-1}$ & $s^{-1}$ & 1100 & 40 \\
\hline$d_{+1}$ & $s^{-1}$ & 20000 & 88 \\
\hline$d_{-2}$ & $s^{-1}$ & 7400 & 5 \\
\hline$d_{+2}$ & $s^{-1}$ & 15000 & 14 \\
\hline$d_{-3}^{+2}$ & $s^{-1}$ & 17600 & 2 \\
\hline$d_{+3}$ & $s^{-1}$ & 2000 & 8 \\
\hline$k_{-1}$ & $s^{-1}$ & 4000 & 50 \\
\hline$k_{+1}$ & $M^{-1} s^{-1}$ & $0.35 \times 10^{6}$ & 25 \\
\hline$k_{-2}$ & $s^{-1}$ & 2080 & 4 \\
\hline$k_{+2}$ & $M^{-1} s^{-1}$ & $30 \times 10^{6}$ & 46 \\
\hline$k_{-3}$ & $s^{-1}$ & 1700 & 7 \\
\hline$k_{+3}$ & $M^{-1} S^{-1}$ & $160 \times 10^{6}$ & 7 \\
\hline$E_{1}$ & & 0.1 & 49 \\
\hline$E_{2}$ & & 12.7 & 1 \\
\hline$E_{3}$ & & 30 & 8 \\
\hline$D_{1}$ & & 10 & 78 \\
\hline$D_{2}$ & & 2.1 & 13 \\
\hline$D_{3}$ & & 0.116 & 8 \\
\hline$K_{1}$ & $\mu \mathrm{M}$ & 14000 & 49 \\
\hline$K_{2}$ & $\mu \mathrm{M}$ & 200 & 84 \\
\hline$K_{3}$ & $\mu \mathrm{M}$ & 10 & 10 \\
\hline$E C_{50}$ & $\mu \mathrm{M}$ & 67 & 5 \\
\hline$n_{\mathrm{H}}$ & & 2.58 & 3 \\
\hline
\end{tabular}

Average rate constants and errors from the fit of scheme 3 (Fig. 3) to our three sets of experiments. Examples of the fits are shown in Figure 7. All 18 rate constants were fitted freely in HJCFIT. Scheme 3 has three equivalent binding sites and three extra shut states that the receptor can access from the resting states. The efficacies, the dissociation equilibrium constants per site, the equilibrium constants for the entry into the extra shut states $\left(D_{n}=d_{+_{n}} / d_{-_{n}}\right)$, the $E C_{50}$, and the Hill slope calculated from the rate constants are listed at the bottom of the table.

nel opening. In the spirit of Monod et al. (1965), this conformation change is concerted, and binding to each conformation is independent. Thus there are only two equilibrium constants for binding, denoted $K_{\mathrm{R}}$ for the resting conformation (R) and $K_{\mathrm{F}}$ for
$1000 \mu \mathrm{M}$
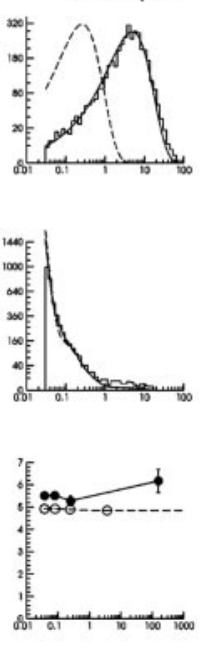

the flipped conformation (F). As a result, this mechanism has 14 free parameters, 4 fewer than the other mechanisms with 3 open and 7 shut states that have been tried so far.

In this mechanism the additional closed states are between the resting state of the receptor and the open states (so they could, for example, correspond to the physical process of domain closure around a bound agonist molecule; see Discussion). Each ligation state of the resting state of the protein $(\mathrm{R})$ can switch to a different closed state $(F)$ while the channel is still shut. It is from these F states that the protein can reach the open state $\left(\mathrm{F}^{*}\right)$. The flip mechanism is appealing in that it provides a physical explanation for how binding sites that are so far apart appear to interact strongly, i.e., how binding to one can affect binding to another, and does so with fewer free parameters than other mechanisms that fit well.

The experimental results were fitted with scheme 5 with the constraint that affinity for the three binding sites remains the same as long as the protein stays in the same conformation ( $\mathrm{R}$ or $\mathrm{F}$ ). The different conformations of the shut protein have the same dissociation constants ( $K_{\mathrm{R}}$ and $K_{\mathrm{F}}$, respectively), regardless of how many other binding sites are already occupied. This is ensured by applying the following constraints while fitting:

$$
\begin{aligned}
& k_{+1}=k_{+2}=k_{+3}\left(=k_{+}\right), \quad k_{-1}=k_{-2}=k_{-3}\left(=k_{-}\right), \\
& k_{\mathrm{F}+2}=k_{\mathrm{F}+3}\left(=k_{\mathrm{F}+}\right), \quad k_{\mathrm{F}-3}=k_{\mathrm{F}-2}\left(=k_{\mathrm{F}-}\right) \text {. }
\end{aligned}
$$

Two rate constants $\left(\gamma_{1}\right.$ and $\left.\gamma_{3}\right)$ (Fig. 3, scheme 5) were constrained to satisfy the requirement of microscopic reversibility for the two cycles in the scheme. As usual, statistical factors are included in the rate constants to take into account the number of available sites at each binding step.

The fit of the flip mechanism, which has 14 free parameters, is shown in Figure 8. The predicted dwell-time (HJC) distributions are in excellent agreement with the data. In particular, the shuttime fast component is predicted extremely well, with no appreciable decrease in quality when compared with the fit of scheme 3 with 18 free parameters. The open-shut correlations are also well accounted for at all concentrations.

This fit provides the best agreement with the data obtained among the schemes that had 14 (or fewer) free parameters and is better than some that had more free parameters. The predicted apparent $P_{\text {open }}$ curve fits quite well, and the predicted Hill slope (at the $E C_{50}$ ) is 2.44 (Table 5), a reasonable value although somewhat smaller than that found from the fit of the Hill equation in Figure 2. It is, of course, the quality of the fit of the whole apparent $P_{\text {open }}$ curve that matters, not just the Hill slope values, and it must be borne in mind that the Hill equation is not a correct description of the data (see section on the $P_{\text {open }}$ curve).

Unfortunately, the quality of the fit (Fig. 8) cannot be distinguished from that produced by scheme 3, with 18 free parameters (Fig. 7). The values of the 14 free rate constants can all be estimated from the fits, as shown by the coefficients of variation (Table 5) and also by the results of simulated fits (see below). The 
pre-opening conformation change to the flipped state is accompanied by an increase in glycine affinity of approximately 65fold $\left(K_{\mathrm{R}}=520 \mu \mathrm{M} ; K_{\mathrm{F}}=8 \mu \mathrm{M}\right)$. Consequently, there is also a 65 -fold increase in $F$ with each extra agonist bound. The first (and biggest) contribution to channel efficacy comes from this increase in $F$. The channel opening equilibrium constants, $E$, also increase as more ligands are bound, but to a smaller extent. The consequences of this two-stage conformation change for the interpretation of efficacy will be considered in the Discussion. It is intriguing that most of the difference in glycine affinity between the resting $\mathrm{R}$ states and the flipped $\mathrm{F}$ states is caused by an increase in the association rate constant. A flip model in which all association rate constants were constrained to be equal (and all the difference in affinity would stem from differences in dissociation rate) gave a much worse description of the dwell-time distributions (data not shown).

We tried fitting scheme 5 (and also schemes 1, 3, and 4 of Fig. 3 ) with the open states connected, thus adding two more free parameters in each scheme. The fits were not detectably improved, and the rates for binding in the open conformation were much smaller than those for binding in the flipped or shut conformation. Thus, if transitions between open states are allowed, they occur rarely and do not need to be included in our schemes.

Ranking of the fits, as judged by the maximum value of the likelihood attained, showed that schemes 3, 4, and 5 produced good fits, whereas schemes 1 (especially with no interaction) and 2 were much worse. The ranking was the same for all three data sets, and it was the same when the Akaike information criterion or the Bayesian information criterion (Stoica et al., 2004) was used rather than likelihood. Scheme 3 was somewhat better than schemes 4 or 5 by all of these criteria, despite the fact that no convincing difference between the quality of the fits in Figures 7 and 8 can be distinguished by eye. Because these mechanisms are not nested, it is not possible to assess in any quantitative way the importance of the differences seen when models are ranked by these criteria. It is also relevant that even for nested models, the distribution of the likelihood ratio may not follow the classic $\chi^{2}$ distribution when some rates are supposed to be zero (i.e., constrained to values on the edge of the parameter space) (Self and Liang, 1987; Celentano and Hawkes, 2004). We therefore conclude that no clear distinction among schemes 3,4 , and 5 can be made at present.

It may be noticed that the flip mechanism, like all others that we have tried, contains no provision for unliganded openings (or for flipping in the absence of agonist). Such states must exist, of course, but because we cannot detect them, there is no point in including them in the mechanism. Nevertheless, at sufficiently low agonist concentrations, binding to preexisting unliganded flip conformation must be the predominant route by which the monoliganded flipped conformation of the receptor (AF) is formed.

In summary, single-channel properties of glycine $\alpha 1 \beta$ receptors are well described both by scheme 3 (with no constraints; 18 18 free parameters).
B
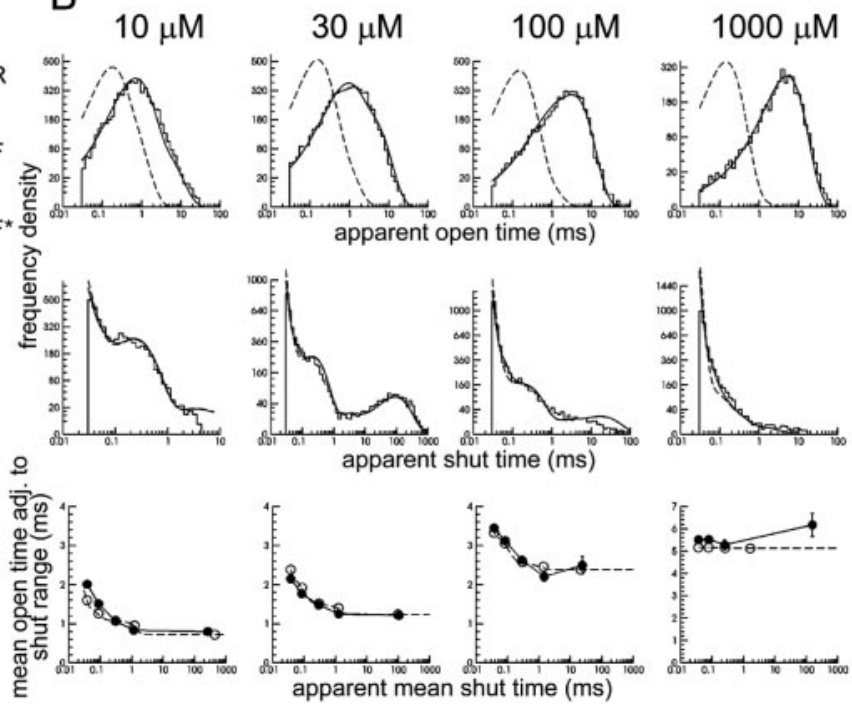

Figure 8. The flip mechanism: three additional shut states resulting from a pre-opening conformational change (scheme 5,14 列 (he rate constants) is indicated in the figure by displaying the same dissociation constants on the binding steps. $B$

Table 5. Estimated parameters from the fit of the flip mechanism to singlechannel data from heteromeric receptors

\begin{tabular}{|c|c|c|c|}
\hline & Unit & Mean estimates & CV (\%) \\
\hline$\alpha_{1}$ & $s^{-1}$ & 3400 & 13 \\
\hline$\beta_{1}$ & $s^{-1}$ & 4200 & 22 \\
\hline$\alpha_{2}$ & $s^{-1}$ & 2100 & 12 \\
\hline$\beta_{2}$ & $s^{-1}$ & 28000 & 17 \\
\hline$\alpha_{3}$ & $s^{-1}$ & 7000 & 18 \\
\hline$\beta_{3}$ & $s^{-1}$ & 129000 & 4 \\
\hline$\gamma_{1}$ & $s^{-1}$ & 29000 & 10 \\
\hline$\delta_{1}$ & $s^{-1}$ & 180 & 24 \\
\hline$\gamma_{2}$ & $s^{-1}$ & 18000 & 14 \\
\hline$\delta_{2}$ & $s^{-1}$ & 6800 & 6 \\
\hline$\gamma_{3}$ & $s^{-1}$ & 900 & 26 \\
\hline$\delta_{3}$ & $s^{-1}$ & 20900 & 3 \\
\hline$k_{-}$ & $s^{-1}$ & 300 & 6 \\
\hline$k_{+}$ & $M^{-1} S^{-1}$ & $0.59 \times 10^{6}$ & 6 \\
\hline$k_{\mathrm{F}-}$ & $s^{-1}$ & 1200 & 12 \\
\hline$k_{\mathrm{F}+}$ & $M^{-1} S^{-1}$ & $150 \times 10^{6}$ & 5 \\
\hline$E_{1}$ & & 1.3 & 21 \\
\hline$E_{2}$ & & 13 & 9 \\
\hline$E_{3}$ & & 20 & 16 \\
\hline$F_{1}$ & & $6 \times 10^{-3}$ & 18 \\
\hline$F_{2}$ & & 0.40 & 15 \\
\hline$F_{3}$ & & 27 & 31 \\
\hline$K_{\mathrm{R}}$ & $\mu \mathrm{M}$ & 520 & 12 \\
\hline$K_{\mathrm{F}}$ & $\mu \mathrm{M}$ & 8 & 14 \\
\hline$E C_{50}$ & $\mu \mathrm{M}$ & 68 & 5 \\
\hline$n_{\mathrm{H}}$ & & 2.44 & 1 \\
\hline
\end{tabular}

Average rate constants and errors from the fit of flip mechanism (scheme 5) (Fig. 3) to our three sets of experiments. Examples of the fits are shown in Figure 8. Scheme 5 has three equivalent binding sites and allows a switch between two different conformational states of the shut receptor. The fits constrain the binding and unbinding rates to be the same, regardless of the number of molecules already bound, in any one conformation. Two rates are set by microscopic reversibility, so there were 14 free parameters. The efficacies, the equilibrium constants for the flip into the $F$ states $\left(F_{n}=\delta_{n} / \gamma_{n}\right)$, the dissociation equilibrium constants per site, the $E C_{50}$, and the Hill slope calculated from the rate constants are listed at the bottom of the table. 


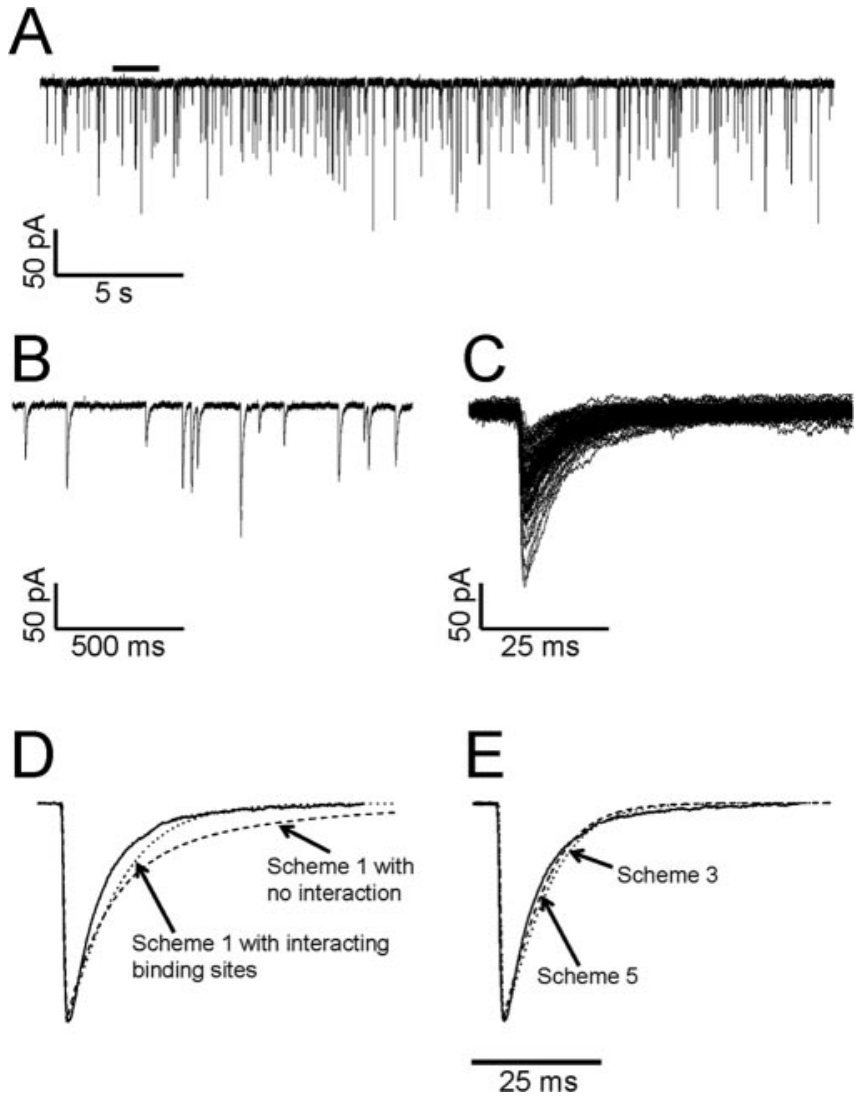

Figure 9. The decay of spontaneous glycinergic IPSCs in rat spinal motoneurons in vitro is well accounted for by activation mechanisms that describe single-channel behavior. A, Spontaneous glycine-mediated synaptic current in a P18 rat motoneuron held at $-70 \mathrm{mV}$; the bar shows portion of the trace shown at an expanded time scale in B.C, IPSCs aligned by their peak. $D, E$, Comparison of the observed IPSC decay with the response to a $1 \mathrm{~ms}$ pulse of $1 \mathrm{~mm}$ glycine, calculated from the results of fitting schemes 1,3, or 5 to the single-channel data. Note that the mechanisms that best describe IPSC time course are the same schemes that best fit singlechannel results: namely, schemes 3 and 5 .

free parameters) and by the flip mechanism (14 free parameters). Apart from the fewer parameters needed, the flip scheme has the advantage that a pre-opening conformational change is physically plausible and provides an attractive explanation for how binding sites that are so far apart appear to interact strongly, i.e., how binding to one can affect binding to another.

\section{Spontaneous glycinergic IPSCs from juvenile rat spinal motoneurons}

The heteromeric $\alpha 1 \beta$ receptor is probably the dominant form of glycine receptor at synapses in adult spinal cord. It is therefore important to test whether the activation schemes that we fitted to single-channel data could also predict the shape of the IPSCs recorded at a real synapse. Spontaneous glycinergic synaptic currents were recorded from motoneurons in spinal slices from P18-P20 animals, an age at which the developmental switch between $\alpha 2$ and $\alpha 1$ is complete (Takahashi et al., 1992; Legendre, 2001). Figure $9 A$ shows a continuous recording from a motoneuron, voltage clamped at $-70 \mathrm{mV}$. Miniature IPSCs are shown on an expanded time scale in Figure 9B. Selected events were aligned by their peak (Fig. 9C) and averaged. The continuous line in Figure $9 D$ shows the normalized average of 240 events. Individual mIPSCs in each cell were averaged, and the decay phase of the average ( $90 \%$ of peak to baseline) was fitted with a single exponential. An attempt to fit the decay with two exponential compo- nents did not produce any significant improvement in the fit: in five of nine cells the second component could not be detected, and in the remaining four it had an amplitude below $5 \%$ of the total. The average value for the decay time constant was $7.5 \pm 0.1$ ms $(n=9$ cells) in accord with reported observations (for instance, $6.3 \mathrm{~ms}$ for spontaneous IPSCs in hypoglossal motoneurons of P10-P18 rats) (Singer et al., 1998).

Our results from the model fitting to the single-channel data were then used to calculate the current response to a $1 \mathrm{~ms}$ concentration jump to $1 \mathrm{~mm}$ glycine. Although the concentration of glycine in the synaptic cleft, and its time course, are still unknown, we chose a jump to $1 \mathrm{~mm}$ glycine lasting for $1 \mathrm{~ms}$ for two reasons. The first is analogy with glutamatergic synapses, where glutamate reaches a concentration of approximately $1 \mathrm{~mm}$ in the cleft (Clements et al., 1992; Tong and Jahr, 1994). Second, the current relaxation after the jump is not very sensitive to changes in concentration (between 1 and $3 \mathrm{~mm}$ ) or in the duration of the pulse (between 1 and $3 \mathrm{~ms}$ ). Figure 9D compares the decay of the observed average mIPSC with that predicted by the two variants of scheme 1, namely with the constraint of no interaction between the binding sites (as in Fig. 4) and with no constraint (as in Fig. 6). It is apparent that both variants predicted a decay time longer than observed. In this particular experiment, the decay of the IPSC was $6.8 \pm 0.1 \mathrm{~ms}$. Fitting the calculated decay of the macroscopic response with a single exponential gave a time constant of $13.1 \mathrm{~ms}$ for scheme 1 with no interaction in binding and a marginally improved $9.6 \mathrm{~ms}$ for scheme 1 with no constraints. On the other hand, the two models that described the singlechannel data accurately were in much better agreement with the observed IPSC decay. As shown in Figure 9E, the decay time for scheme 3 and for the flip mechanism (scheme 5) were 7.6 and 6.9 $\mathrm{ms}$, respectively, which is very close to the observed values. The time course of the macroscopic current, calculated from the fit, has $k-1$ exponential components (six components for scheme 1 and nine for schemes 3 and 5) (Colquhoun and Hawkes, 1977), but for a jump to zero concentration only one component was found to have any substantial amplitude, and the time constant for that is given. This is in agreement with the detection of only one component in the decay of mIPSCs. The decay time constant of the IPSC is close to the slowest component in the burst length distribution predicted by both scheme 3 and the flip scheme at very low agonist concentrations (approximately $6 \mathrm{~ms}$ for both). This is expected on theoretical grounds (Colquhoun et al., 1997; Wyllie et al., 1998).

The decay time of IPSCs is known to be influenced by several factors, including the distance of the synapse from the recording site and electrotonic filtering, so the remarkable agreement of the predicted decay derived from single-channel measurements with the observed decay time must be viewed with caution.

\section{Reanalysis of results with homomeric $\alpha 1$ receptors with the flip mechanism}

We showed recently (Beato et al., 2004) that the $\alpha 1$ homomeric glycine receptor is described quite accurately by a scheme with three binding sites that interact in the shut conformation. In that case, the estimates of $K_{1}=3200 \mu \mathrm{M}, K_{2}=110 \mu \mathrm{M}$, and $K_{3}=420$ $\mu \mathrm{M}$ did not decrease as regularly as found here with the heteromeric receptor (Table 3 ), so it was not so obvious that a flip mechanism might account for the results. Given the success of the flip mechanism in explaining the interaction between binding sites for the heteromeric receptor and the qualitatively similar interactions seen for the homomeric receptor, we have refitted the previously published data from the homomeric glycine recep- 
tor (Beato et al., 2004), now using the flip mechanism. Six sets of experiments at four different concentrations of glycine $(10,50$, 100 , and $1000 \mu \mathrm{M})$ were simultaneously fitted with scheme 5 , and the results are reported in Figure $10 \mathrm{~B}$. The HJC distributions are all in good agreement with the data, and the fits are virtually indistinguishable from those previously reported, despite the fact that the flip mechanism constrains the apparent affinity for subsequent bindings to increase monotonically. $P_{\text {open }}$ data are also well fitted, giving an $E C_{50}$ of $109 \mu \mathrm{M}$, a Hill slope of 2.0, and a maximum $P_{\text {open }}$ of 0.97 , compared with the experimental values of $106 \mu \mathrm{M}, 1.8$, and 0.98 , respectively. The average rates are reported in Table 6 . As expected, the flipped conformation has a higher affinity for glycine than the resting conformation, but the increase is only 6-fold (compared with 65 -fold for the heteromeric receptor). This is not unexpected in the light of the shallower Hill slope found for the homomeric receptor. When the same homomeric data were fitted with a scheme with five binding sites and the addition of flipped states before the open states (as in scheme 5), the singlechannel distributions were well fitted (data not shown); however, such a scheme with five binding sites predicted a Hill slope of 2.5, which is much steeper than the value (1.8) found for the homomeric receptor by fitting the Hill equation. Attempts to use five binding sites while constraining the gating rate constants to be equal after the third binding (Beato et al., 2004) did not give satisfactory fits of the single-channel distributions (data not shown), although this model correctly predicted the Hill slope (2.0).

\section{Simulations}

Table 5 shows that the estimate for the opening rate constant, $\beta_{3}$, for the flip mechanism is very fast, $\sim 130,000 \mathrm{~s}^{-1}$, for the heteromeric receptor. At first we were deeply skeptical about our ability to estimate such a fast value from data with a resolution of $30 \mu \mathrm{s}$. Although it is true that the errors in the rate constant estimates reported by HJCFIT (found from the curvature of the likelihood surface at its maximum) are all tolerably small and the values are quite reproducible for the three independent data sets, it seemed advisable to test estimates by doing repeated fits to simulated data [as reported for nicotinic receptor fits by Colquhoun et al. (2003)]. Repeated sets of "experimental" data were simulated using a random number generator, as in Colquhoun et al. (2003). The flip mechanism was used, and the estimates of the rate constants in Table 5 were taken as the true values (Table 7, column 3 ). Each "experiment" consisted of a set of four simulated records (lists of open and shut times) that, after imposition of a resolution of $30 \mu \mathrm{s}$, had approximately 15,000 transitions, as in the experiments. The large number of missed events can be judged by the fact that to get 15,000 resolved transitions, it was necessary to generate $58,000,120,000,240,000$, and 400,000 transitions at 10, 30,100 , and $1000 \mu \mathrm{M}$ glycine, respectively.

Two questions can be addressed by simulated fits: (1) how good are the estimates of rate constants, and (2) what happens if the wrong mechanism is fitted?

The question of the quality of estimates is examined by inspec-
B
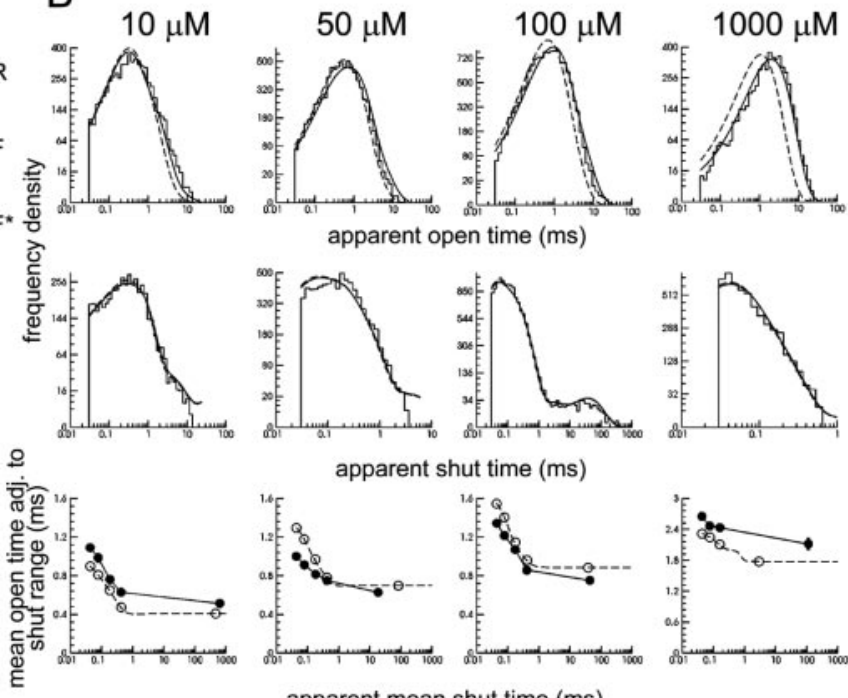

apparent shut time (ms)
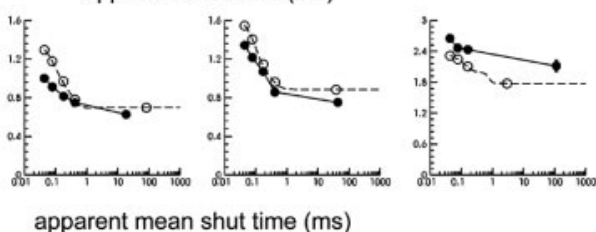

apparent mean shut time (ms)

Figure 10. Test of the flip mechanism on data from homomeric $\alpha 1$ glycine receptors. $A$, Scheme 5 , with binding rates 政 e 5 provides an excellent prediction of single-channel behavior for $\alpha 1$ homomeric glycine receptors. The agreement of scheme 5 with the data is of a quality comparable with that observed for the best-fitting models in Beato et al. (2004), which were scheme 1 with no constraints or an extended scheme 1, with five binding sites and saturation of gating.

tion of the sampling distribution of our estimator. Examples of these are shown in Figure 11. The first six panels in Figure 11 are examples of the worst distributions obtained when bad initial guesses were used for the fits (Table 7 , guess 1). These guesses differed from the true values by factors of 1.3-14, the factor being larger than 5 for 8 of the 14 free parameters. In particular the guess for the fastest rate constant, $\beta_{3}=130,000 \mathrm{~s}^{-1}$ was only $15,000 \mathrm{~s}^{-1}$. Although a majority of fits were good, 78 of 257 (30\%) of fits gave values of $\beta_{3}$ and $\alpha_{3}$ that were too big, and the same 78 fits also gave estimates of $k_{\mathrm{F}-}$ that were too small. These are seen as separate peaks in Figure $11 A-C$. When the 78 fits that gave $\alpha_{3}>10,000$ are excluded (Fig. $11 D, E$ ), excellent estimates are obtained. Figure $11 \mathrm{~F}$ shows the maximum likelihood attained in each fit, which is seen to be essentially the same for fits in which $\alpha_{3}$ was in the right peak or the high peak. Therefore, this is clearly another instance of the existence of two solutions to the missed event problem (Ball et al., 1990, 1994; Colquhoun and Hawkes, 1995). The results are much like those described by Colquhoun et al. (2003) with a different mechanism, although the problem is less serious in this case, because the "slow" and "fast" solutions are sufficiently close that the difference between them would not matter greatly in practice.

When better guesses were used (Table 7, guess 2) the wrong (fast) solution was found very rarely. Examples of the results of 576 fits that started from guess 2 are shown in Figure $11 G-L$, and the results are shown in Table 7 . Only four of the 576 fits gave aberrant values, and these have been excluded in Figure 11, G and $H$. All 576 fits are shown for the association and dissociation rate constants in Figure $11 \mathrm{I}-\mathrm{L}$. All of these estimates are seen to be good, as are those of the rest of the 14 free parameters. Table 7 shows that the coefficient of variation for the estimates ranged from 1.5 to $14.2 \%$, with the very fast opening rate constant $\left(\beta_{3}=\right.$ $\left.130,000 \mathrm{~s}^{-1}\right)$, perhaps surprisingly, being the most precise of all as well as being one of the least biased estimates $(0.74 \%)$. This was true despite a positive strong correlation between the estimates of $\beta_{3}$ and $\alpha_{3}$, similar to that seen by Colquhoun et al. (2003). The 
Table 6. Estimated parameters from the fit of the flip mechanism to singlechannel data from homomeric $\alpha 1$ receptor

\begin{tabular}{|c|c|c|c|}
\hline & Unit & Mean estimates & CV (\%) \\
\hline$\alpha_{1}$ & $s^{-1}$ & 4100 & 2 \\
\hline$\beta_{1}$ & $s^{-1}$ & 1500 & 6 \\
\hline$\alpha_{2}$ & $s^{-1}$ & 1400 & 4 \\
\hline$\beta_{2}$ & $s^{-1}$ & 18000 & 5 \\
\hline$\alpha_{3}$ & $s^{-1}$ & 700 & 3 \\
\hline$\beta_{3}$ & $s^{-1}$ & 28000 & 5 \\
\hline$\gamma_{1}$ & $s^{-1}$ & 500 & 1 \\
\hline$\delta_{1}$ & $s^{-1}$ & 100 & 5 \\
\hline$\gamma_{2}$ & $s^{-1}$ & 11500 & 6 \\
\hline$\delta_{2}$ & $s^{-1}$ & 15500 & 8 \\
\hline$\gamma_{3}$ & $s^{-1}$ & 1600 & 12 \\
\hline$\delta_{3}$ & $s^{-1}$ & 13000 & 7 \\
\hline$k_{-}$ & $s^{-1}$ & 200 & 7 \\
\hline$k_{+}$ & $M^{-1} S^{-1}$ & $0.23 \times 10^{6}$ & 6 \\
\hline$k_{\mathrm{F}-}$ & $s^{-1}$ & 1500 & 11 \\
\hline$k_{\mathrm{F}+}$ & $M^{-1} S^{-1}$ & $11 \times 10^{6}$ & 9 \\
\hline$E_{1}$ & & 0.4 & 5 \\
\hline$E_{2}$ & & 12 & 5 \\
\hline$E_{3}$ & & 38 & 5 \\
\hline$F_{1}$ & & 0.2 & 7 \\
\hline$F_{2}$ & & 1 & 7 \\
\hline$F_{3}$ & & 8 & 11 \\
\hline$K_{\mathrm{R}}$ & $\mu \mathrm{M}$ & 900 & 6 \\
\hline$K_{\mathrm{F}}$ & $\mu \mathrm{M}$ & 150 & 5 \\
\hline$E C_{50}$ & $\mu \mathrm{M}$ & 109 & 4 \\
\hline$n_{\mathrm{H}}$ & & 2.0 & 1 \\
\hline
\end{tabular}

Six sets of cell-attached data for the homomeric $\alpha 1$ receptors were fitted with scheme 5 (Fig. 3). The average of the rates for each set is reported in this table. Examples of the fits are shown in Figure 10. The difference in affinity between the shut and flipped states is much reduced compared with the heteromer. This is reflected in the shallower Hill slope. least precise estimates were for the monoliganded flip rates, $\gamma_{1}$ and $\delta_{1}$, both of which have a CV of approximately $13 \%$, although the $\mathrm{CV}$ of their ratio, the equilibrium constant $F_{1}$, was smaller $(7.1 \%)$ as a result of the positive correlation $(r=0.85)$ between the estimates of these two rate constants. As is apparent from Figure 11, many of the estimates were biased to a noticeable extent $(-5.2$ to $+11 \%)$ (Table 7$)$, although not enough to be of any importance in practice. The largest bias is in the estimate of $k_{+}$, and this is shown in Figure $11 \mathrm{~L}$. There is a strong positive correlation between the estimates of $k_{+}$and $k_{-}(r=0.92)$, which is why their ratio, $K_{\mathrm{R}}$, is more precise $(\mathrm{CV}=1.9 \%)$ than either rate constant.

In summary, we can get good estimates of all 14 rate constants from our experiments. Of course there is an element of circularity in any simulation study. To be more precise, all we can say is that if the true values were as shown in Table 7 , then we would be able to get good estimates of all of them from the sort of experiments that were done.

Simulations were used also to investigate the extent to which it was possible to discriminate between the two best-fitting mechanisms, scheme 3 with 18 free parameters, and the flip mechanism (scheme 5) with 14 free parameters. When data were simulated from the flip mechanism but then fitted with scheme 3 , the fits were essentially perfect by all the criteria that we usually use. When data were simulated with scheme 3 , but then fitted with the flip mechanism, again good fits were obtained; there were small but systematic signs of imperfect fit of the shut-time distribution, especially at $30 \mu \mathrm{M}$ glycine, but it is doubtful whether they were big enough to be detected reliably in a real experiment.

\section{Discussion}

Some things are clear about the activation mechanism of heteromeric $\alpha 1 \beta$ glycine receptors. We attempted to fit 30 different reaction schemes (of which 9 are reported here) to the steadystate single-channel activity evoked by glycine concentrations

Table 7. Repeated fits of the flip mechanism to simulated data

\begin{tabular}{|c|c|c|c|c|c|c|c|}
\hline & Unit & True & Guess 1 & Guess 2 & Mean & CV (\%) & Bias \\
\hline$\alpha_{1}$ & $s^{-1}$ & 3400 & 5000 & 5000 & 3416 & 4.6 & 0.48 \\
\hline$\beta_{1}$ & $s^{-1}$ & 4200 & 500 & 8000 & 4178 & 10.6 & -0.54 \\
\hline $\begin{array}{l}\alpha_{1} \\
\alpha_{2}\end{array}$ & $s^{-1}$ & 2100 & 2700 & 2700 & 2087 & 3.6 & -0.61 \\
\hline$\beta_{2}$ & $s^{-1}$ & 28000 & 2000 & 20000 & 27247 & 7.3 & -2.7 \\
\hline$\alpha_{3}$ & $s^{-1}$ & 6700 & 800 & 4000 & $7010^{a}$ & 6.1 & 4.6 \\
\hline$\beta_{3}$ & $s^{-1}$ & 130000 & 15000 & 160000 & $130966^{a}$ & 1.5 & 0.74 \\
\hline $\begin{array}{l}\gamma_{3} \\
\gamma_{1}\end{array}$ & $s^{-1}$ & $29266^{b}$ & 7500 & 6250 & 29267 & 13.1 & 5.0 \\
\hline$\delta_{1}^{\gamma_{1}}$ & $s^{-1}$ & 180 & 1200 & 100 & 194.3 & 13.0 & 8.0 \\
\hline$\gamma_{2}$ & $s^{-1}$ & 18000 & 1500 & 10000 & 17848 & 3.9 & -0.84 \\
\hline$\delta_{2}$ & $s^{-1}$ & 6800 & 12000 & 4000 & 6851 & 2.5 & 0.75 \\
\hline $\begin{array}{l}\gamma_{3} \\
\gamma_{3}\end{array}$ & $s^{-1}$ & $948.1^{b}$ & 300 & 3000 & 897 & 14.2 & -5.4 \\
\hline$\delta_{3}$ & $s^{-1}$ & 22000 & 120000 & 30000 & 20788 & 10.1 & -5.1 \\
\hline$k_{-}$ & $s^{-1}$ & 302 & 1000 & 500 & 317 & 4.7 & 4.9 \\
\hline$k_{+}$ & $M^{-1} s^{-1}$ & $0.59 \times 10^{6}$ & $4.5 \times 10^{6}$ & $1.0 \times 10^{6}$ & $0.65 \times 10^{6}$ & 5.0 & 11.1 \\
\hline$k_{\mathrm{F}-}$ & $s^{-1}$ & 1250 & 2000 & 2000 & 1185 & 5.8 & -5.2 \\
\hline $\begin{array}{l}\mathrm{F}_{-}- \\
k_{\mathrm{F}+}\end{array}$ & $M^{-1} s^{-1}$ & $150 \times 10^{6}$ & $450 \times 10^{6}$ & $100 \times 10^{6}$ & $149 \times 10^{6}$ & 5.0 & -0.7 \\
\hline$E_{1}$ & & 1.23 & & & 1.225 & 11.5 & -0.81 \\
\hline$E_{2}$ & & 13.3 & & & 13.0 & 4.4 & -2.2 \\
\hline$E_{3}$ & & 19.4 & & & $18.7^{a}$ & 4.8 & 3.4 \\
\hline$F_{1}$ & & 0.00615 & & & 0.00634 & 7.2 & 3.1 \\
\hline$F_{2}$ & & 0.380 & & & 0.384 & 1.1 & 1.7 \\
\hline$F_{3}$ & & 23.2 & & & 23.4 & 6.7 & 0.74 \\
\hline$K_{\mathrm{B}}^{3}$ & $\mu \mathrm{M}$ & 512 & & & 483 & 1.95 & -5.6 \\
\hline$K_{\mathrm{F}}^{\mathrm{n}}$ & $\mu \mathrm{M}$ & 8.33 & & & 7.96 & 5.5 & -4.4 \\
\hline
\end{tabular}

The column labeled "true" shows the values of the rate constants that were used for simulations of the experiments, based on the flip mechanism (scheme 5 ), and fitted with the same mechanism (14 free rate constants). Figure $11 A-F$ shows examples of the distributions of rate constant estimates from 257 fits that started from the bad guesses given in the column labeled "guess 1 ." Figure $11 \mathrm{G}-L$ shows examples from 576 fits that started from the better guesses given in the column labeled "guess 2 ." The last three columns of the table give the mean, $(V$, and bias of the estimates of each rate constant for fits that started from guess 2 ( 6 of the 14 distributions are shown in Fig. $11 \mathrm{G}-L$ ). 
A

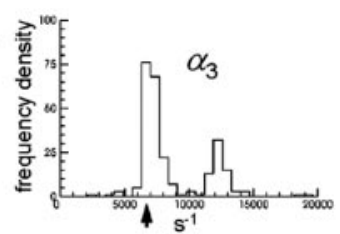

$\mathrm{C}$

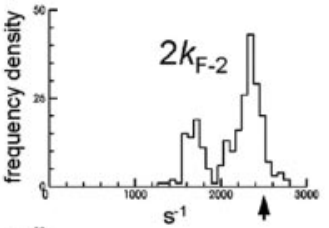

E

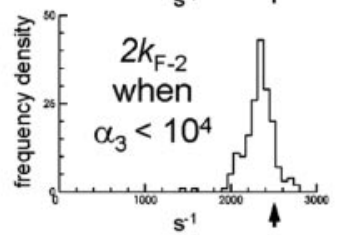

G

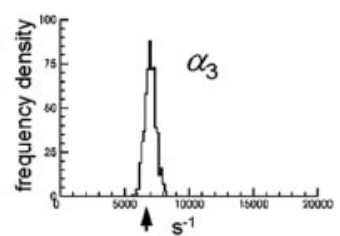

I

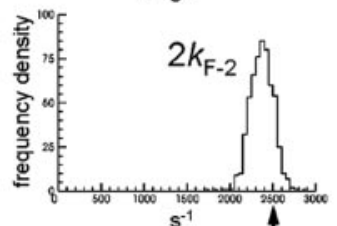

$\mathrm{K}$

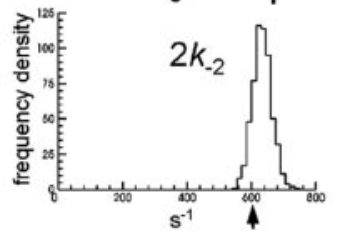

B

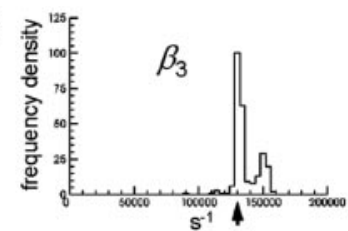

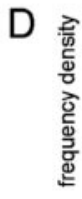

$\mathrm{F}$
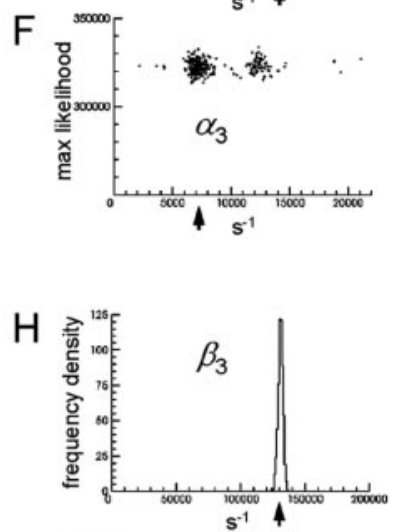

$\mathrm{J}$

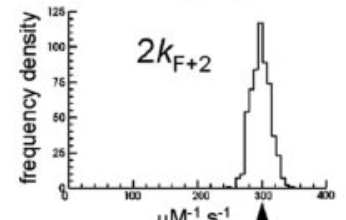

$\mathrm{L}$

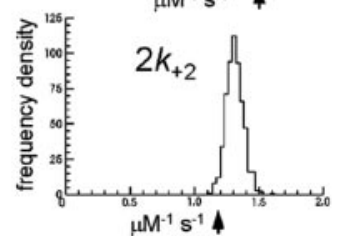

Figure 11. Results of repeated fits to simulated data sets. Results were simulated with the flip mechanism (scheme 5), a resolution of $30 \mu$ s was imposed, and "data" for 10, 30, 100, and $1000 \mu \mathrm{m}$ were fitted simultaneously with the same mechanism (14 free rate constants). The first six graphs $(A-F)$ are from 257 fits that started from bad guesses (Table 7, guess 1 ). The last six graphs ( $G-L$ ) are from 576 fits that started from better guesses (Table 7, guess 2 ). All graphs apart from $F$ show the distributions of the estimates of a rate constant that were obtained in each fit. The arrows show the true value of that rate constant. $\ln F$, the maximum likelihood found in each fit is plotted against the value of $\alpha_{3}$ found in the same fit: the two clusters of points correspond to the two peaks in $A$.

from 10 to $1000 \mu \mathrm{M}$. To get good fits, mechanisms had to (1) have three glycine binding sites, (2) allow channel opening from both partially liganded and fully liganded receptors, with efficacy increasing with the level of ligation, (3) contain more shut states than the four required by a simple sequential scheme, (4) account for open-shut correlations (i.e., have no single gateway state) (Fredkin et al., 1985), and (5) appear to show interaction between binding sites while the channel is still shut. One important thing is still not clear: we cannot distinguish between the two bestfitting mechanisms (Fig. 12), and these two mechanisms have different implications for structural changes.

\section{Mechanism versus description}

Scheme 3 (Fig. 12B) is an extension of the model used by Jones and Westbrook (1995) to describe $\mathrm{GABA}_{\mathrm{A}}$ macroscopic currents.

A

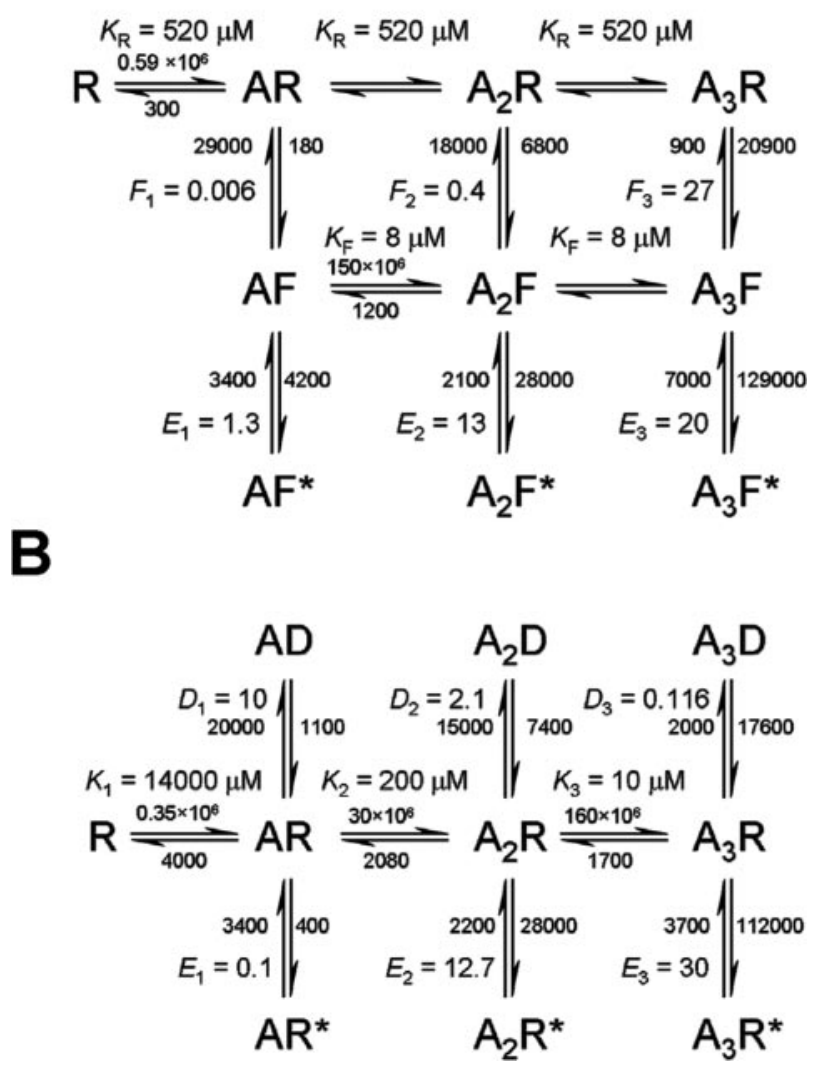

Figure 12. A comparison of the flip mechanism (scheme 5) $(A)$ with scheme $3(B)$. The averaged rate constants (units as in Tables 4 and 5 ) and equilibrium constants are shown. Both mechanisms gave good fits with these values (Figs. 7, 8).

When fitting scheme 3 , it was found consistently that in the shut receptor, binding of the second molecule of glycine appeared to have higher affinity than the first, and the third had higher affinity than the second. The third binding to the shut channel has an equilibrium constant of $10 \mu \mathrm{M}, 1400$ times tighter than the first binding $\left(K_{1}=14 \mathrm{mM}\right)$. This means that a binding site can "sense" whether other binding sites are occupied while the channel is still shut, i.e., that the sites interact, despite being quite a long distance apart. Scheme 3 (Fig. 12 B) provides no explanation of how this interaction occurs; it is just an observation, like the observation that the affinity of hemoglobin for oxygen appears to increase as more oxygen binds when results are fitted with the Adair equation (Wyman and Gill, 1990).

Wyman and Allen (1951) proposed a mechanism for this apparent cooperativity of binding, a mechanism subsequently applied to enzymes (Monod et al., 1965). Wyman and Allen (1951) suggested that if a protein existed in two conformations, one of which had a higher affinity for a ligand than the other, then the ligand would cause a shift to the higher affinity form. This would give the appearance of increasing overall affinity as more ligand binds, even if there were no interaction between the binding sites, i.e., if all of the sites on each conformation were independent and so had the same affinity for ligand, regardless of the number of other sites that were already occupied. The flip mechanism (Fig. $12 \mathrm{~A}$, scheme 5) incorporates this idea. The glycine affinity of all sites depends not on ligation but on what state the receptor is in. The flipped states have an agonist affinity $\left(K_{\mathrm{F}}\right) 65$-fold higher than the resting states $\left(K_{\mathrm{R}}\right)$. Because there are only two equilib- 
rium constants for binding, this mechanism has 14 free parameters, 4 fewer than scheme 3 (Fig. 12 B). In the flip mechanism (Fig. $12 \mathrm{~A}$ ), the conformation changes, both from resting to "flipped" and from flipped to open, are supposed to be concerted, i.e., all five subunits move together.

The homomeric $\alpha 1$ receptor also showed "cooperativity in binding” (Beato et al., 2004), although it was not as strong or as obviously monotonic as found here for heteromers. Nonetheless, we now find that our homomeric data can also be fitted by the flip scheme (Fig. 10). The fit is as good as the sequential mechanism with three interacting binding sites. The increase in affinity after flipping was smaller than for the heteromer (6-fold vs 65-fold), in agreement with the smaller apparent cooperativity of homomeric responses.

Both mechanisms in Figure 12 fit equally well our results on heteromeric receptors, namely single-channel data at a wide range of concentrations (Figs. 7, 8) as well as the decay of spontaneous IPSCs in the spinal cord (Fig. 9), which are likely to be mediated by heteromeric glycine receptors. At present we cannot distinguish between these two mechanisms but to do so is an important job for the future, for three main reasons. First, the flip mechanism implies a rather different way of looking at the binding-gating problem (Colquhoun, 1998). The second reason is that the flip mechanism has important implications for our understanding of the structural dynamics of receptor activation. Third, if the wrong mechanism were fitted, wrong conclusions about binding and gating could result; e.g., a mutation might appear to affect binding when in fact it did not.

\section{Implications of an additional gating step}

del Castillo and Katz (1957) first proposed that it was necessary (if only to explain partial agonism) to consider separately the binding and gating steps. Ever since then, gating has been thought of as a single step. In the flip mechanism, gating is separated into two steps, and the maximum response $\left(P_{\text {open }}\right)$ depends on both of them, being given by $F_{3} E_{3} /\left(1+F_{3}+F_{3} E_{3}\right)$. In Figure $12 A$, it can be seen that both steps contribute to the increase in efficacy as more ligands are bound, but it is the change in flipping $\left(F_{1}\right.$ to $\left.F_{3}\right)$ that contributes the most.

The structural interpretation of the flip mechanism is of great interest. The flipping reaction is a change of shape that precedes channel opening. It is tempting to equate this with the "domain closure" seen in cysteine accessibility data and in structural studies (Gouaux, 2004; Lester et al., 2004), although it is impossible to tell whether structures measured in the presence of agonist are flipped states, open states, or desensitized states. Our results suggest that the increased agonist affinity of the flipped conformation results from an increased association rate rather than a decreased dissociation rate (forcing the change to be only in the dissociation rate gives poor quality fits). Closure of the structure around an agonist would, prima facie, be expected to decrease the dissociation rate (and also the association rate if access became more difficult). Interpretation of flipping as domain closure is sustainable only if the pre-opening conformation change increases the accessibility of the agonist for its binding site, so more collisions result in successful docking and hence higher affinity.

An interesting aspect of both of the best fits (Fig. 12) is that the true mean open time of the channel appears to be much the same regardless of the number of ligands bound $\left(\alpha_{1}, \alpha_{2}\right.$, and $\alpha_{3}$ are similar). This suggests that the stability of the open state is much the same regardless of how many ligands are bound, whereas the stability of the pre-open flipped conformation, and therefore its tendency to open, changes strongly when more ligands are bound. Although long openings appear to be more common at higher con- centrations (Fig. 1), this seems to be attributable primarily to the inability to detect short shuttings. From the rate constants in Figure $12 \mathrm{~A}$, we see that monoliganded openings will usually occur singly, and when more than one occurs in a burst they will be separated by a shut time that is at least $29 \mu$ s on average (the mean lifetime of the AF state at $10 \mu \mathrm{M}$ glycine) and therefore stands a good chance of being detected. In contrast, triliganded openings will occur in bursts of 60 or so openings, separated by shut times that mostly have a mean length of $8 \mu \mathrm{s}$. With a resolution of $30 \mu \mathrm{s}$, only approximately $2 \%$ of these shuttings will be detected, so triliganded openings will appear to be much longer than they really are. A qualitatively similar but less pronounced tendency is seen also for the homomeric receptor (Fig. 10, Table 6).

The problem of whether related mechanisms can be distinguished raises interesting questions of theory (Kienker, 1989). These problems will be the subject of future work, but preliminary results suggest that experiments simulated with the flip mechanism can be fitted very well with scheme 3 (18 free parameters) and that results simulated with scheme 3 can be fitted with the flip mechanism (14 free parameters) in a way that is not perfect but is sufficiently good that it would not be distinguishable in practice, with the sort of recordings that we have made. Possible ways of distinguishing between the rival mechanisms include looking at a range of agonists (in progress), recording single channels after a concentration jump, increasing resolution by using ultra-low noise methods, decreasing temperature, or using the slow $\alpha 2$ subunit (Mangin et al., 2003).

Although distinguishability of mechanisms is a problem, it seems, perhaps surprisingly, that estimatability of rate constants is not. Simulation of repeated fits showed that our data, with the HJCFIT algorithm, are capable of making good estimates of all 14 free rate constants in the flip mechanism, including the opening rate of the fully liganded receptor, $\beta_{3}$, which, at approximately $130,000 \mathrm{~s}^{-1}$, is approximately twice as fast as for a nicotinic receptor (Salamone et al., 1999; Hatton et al., 2003).

In summary, we propose a novel flip mechanism, which is appealing because it provides a physical explanation for apparent site interaction and has the potential for structural interpretation, but we cannot yet distinguish unambiguously between the flip mechanism and some others.

\section{References}

Ball FG, Davies SS, Sansom MS (1990) Single-channel data and missed events: analysis of a two-state Markov model. Proc R Soc Lond B Biol Sci 242:61-67.

Ball FG, Davies SS, Sansom MS (1994) Ion channel gating and time interval omission: statistical inference for a two-state Markov model. Proc R Soc Lond B Biol Sci 255:267-272.

Beato M, Groot-Kormelink PJ, Colquhoun D, Sivilotti LG (2002) Openings of the rat recombinant $\alpha 1$ homomeric glycine receptor as a function of the number of agonist molecules bound. J Gen Physiol 119:443-466.

Beato M, Groot-Kormelink PJ, Colquhoun D, Sivilotti LG (2004) The activation of $\alpha 1$ homomeric glycine receptors. J Neurosci 24:895-906.

Blatz AL, Magleby KL (1989) Adjacent interval analysis distinguishes among gating mechanisms for the fast chloride channel from rat skeletal muscle. J Physiol (Lond) 410:561-585.

Bormann J, Rundström N, Betz H, Langosch D (1993) Residues within transmembrane segment M2 determine chloride conductance of glycine receptor homo- and hetero-oligomers. EMBO J 12:3729-3737.

Brejc K, van Dijk WJ, Klaassen RV, Schuurmans M, van der Oost J, Smit AB, Sixma TK (2001) Crystal structure of an ACh-binding protein reveals the ligand-binding domain of nicotinic receptors. Nature 411:269-276.

Burzomato V, Groot-Kormelink PJ, Sivilotti LG, Beato M (2003) Stoichiometry of recombinant heteromeric glycine receptors revealed by a porelining region point mutation. Receptors Channels 9:353-361.

Celentano JJ, Hawkes AG (2004) Use of the covariance matrix in directly fitting kinetic parameters: application to $\mathrm{GABA}_{\mathrm{A}}$ receptors. Biophys J 87:276-294. Celie PH, Rossum-Fikkert SE, van Dijk WJ, Brejc K, Smit AB, Sixma TK 
(2004) Nicotine and carbamylcholine binding to nicotinic acetylcholine receptors as studied in AChBP crystal structures. Neuron 41:907-914.

Chakrapani S, Bailey TD, Auerbach A (2004) Gating dynamics of the acetylcholine receptor extracellular domain. J Gen Physiol 123:341-356.

Clements JD, Lester RAJ, Tong G, Jahr CE, Westbrook GL (1992) The time course of glutamate in the synaptic cleft. Science 258:1498-1501.

Colquhoun D (1998) Binding, gating, affinity and efficacy: the interpretation of structure-activity relationships for agonists and of the effects of mutating receptors. Br J Pharmacol 125:923-947.

Colquhoun D, Hawkes AG (1977) Relaxation and fluctuations of membrane currents that flow through drug-operated channels. Proc R Soc Lond B Biol Sci 199:231-262.

Colquhoun D, Hawkes AG (1982) On the stochastic properties of bursts of single ion channel openings and of clusters of bursts. Philos Trans R Soc Lond B Biol Sci 300:1-59.

Colquhoun D, Hawkes AG (1987) A note on correlations in single ion channel records. Proc R Soc Lond B Biol Sci 230:15-52.

Colquhoun D, Hawkes AG (1990) Stochastic properties of ion channel openings and bursts in a membrane patch that contains two channels: evidence concerning the number of channels present when a record containing only single openings is observed. Proc R Soc Lond B Biol Sci 240:453-477.

Colquhoun D, Hawkes AG (1995) The principles of the stochastic interpretation of ion-channel mechanisms. In: Single-channel recording (Sakmann B, Neher E, eds), pp 397-482. New York: Plenum.

Colquhoun D, Ogden DC (1988) Activation of ion channels in the frog endplate by high concentrations of acetylcholine. J Physiol (Lond) 395:131-159.

Colquhoun D, Sakmann B (1985) Fast events in single-channel currents activated by acetylcholine and its analogues at the frog muscle end-plate. J Physiol (Lond) 369:501-557.

Colquhoun D, Sigworth FJ (1995) Fitting and statistical analysis of singlechannel records. In: Single-channel recording (Sakmann B, Neher E, eds), pp 483-587. New York: Plenum.

Colquhoun D, Hawkes AG, Srodzinski K (1996) Joint distributions of apparent open and shut times of single-ion channels and maximum likelihood fitting of mechanisms. Philos Trans R Soc Lond A 354:2555-2590.

Colquhoun D, Hawkes AG, Merlushkin A, Edmonds B (1997) Properties of single ion channel currents elicited by a pulse of agonist concentration or voltage. Philos Trans R Soc Lond A 355:1-44.

Colquhoun D, Hatton CJ, Hawkes AG (2003) The quality of maximum likelihood estimates of ion channel rate constants. J Physiol (Lond) 547:699-728.

Colquhoun D, Dowsland KA, Beato M, Plested AJ (2004) How to impose microscopic reversibility in complex reaction mechanisms. Biophys J 86:3510-3518.

del Castillo J, Katz B (1957) Interaction at end-plate receptors between different choline derivatives. Proc R Soc Lond B Biol Sci 146:369-381.

Fredkin DR, Montal M, Rice JA (1985) Identification of aggregated Markovian models: application to the nicotinic acetylcholine receptor. In: Procedures of the Berkeley conference in honor of Jerzy Neyman and Jack Kiefer (Le Cam LM, Olshen RA, eds), pp 269-289. Monterey: Wadsworth.

Gouaux E (2004) Structure and function of AMPA receptors. J Physiol (Lond) 554:249-253.

Groot-Kormelink PJ, Beato M, Finotti C, Harvey RJ, Sivilotti LG (2002) Achieving optimal expression for single channel recording: a plasmid ratio approach to the expression of $\alpha 1$ glycine receptors in HEK293 cells. J Neurosci Methods 113:207-214.

Grosman C, Zhou M, Auerbach A (2000) Mapping the conformational wave of acetylcholine receptor channel gating. Nature 403:773-776.

Handford CA, Lynch JW, Baker E, Webb GC, Ford JH, Sutherland GR, Schofield PR (1996) The human glycine receptor $\beta$ subunit: primary structure, functional characterisation and chromosomal localisation of the human and murine genes. Mol Brain Res 35:211-219.

Hatton CJ, Shelley C, Brydson M, Beeson D, Colquhoun D (2003) Properties of the human muscle nicotinic receptor, and of the slow-channel myasthenic syndrome mutant $\epsilon \mathrm{L} 221 \mathrm{~F}$, inferred from maximum likelihood fits. J Physiol (Lond) 547:729-760.

Hawkes AG, Jalali A, Colquhoun D (1990) The distributions of the apparent open times and shut times in a single channel record when brief events can not be detected. Philos Trans R Soc Lond A 332:511-538.

Hawkes AG, Jalali A, Colquhoun D (1992) Asymptotic distributions of apparent open times and shut times in a single channel record allowing for the omission of brief events. Philos Trans R Soc Lond B Biol Sci 337:383-404.

Jackson MB, Wong BS, Morris CE, Lecar H, Christian CN (1983) Successive openings of the same acetylcholine receptor channel are correlated in open time. Biophys J 42:109-114.

Jones MV, Westbrook GL (1995) Desensitized states prolong $\mathrm{GABA}_{\mathrm{A}}$ channel responses to brief agonist pulses. Neuron 15:181-191.

Kienker P (1989) Equivalence of aggregated Markov models of ion-channel gating. Proc R Soc Lond B Biol Sci 236:269-309.

Kuhse J, Laube B, Magalei D, Betz H (1993) Assembly of the inhibitory glycine receptor: identification of amino acid sequence motifs governing subunit stoichiometry. Neuron 11:1049-1056.

Laube B, Maksay G, Schemm R, Betz H (2002) Modulation of glycine receptor function: a novel approach for therapeutic intervention at inhibitory synapses. Trends Pharmacol Sci 23:519-527.

Legendre P (1998) A reluctant gating mode of glycine receptor channels determines the time course of inhibitory miniature synaptic events in zebrafish hindbrain neurons. J Neurosci 18:2856-2870.

Legendre P (2001) The glycinergic inhibitory synapse. Cell Mol Life Sci 58:560-593.

Lester HA, Dibas MI, Dahan DS, Leite JF, Dougherty DA (2004) Cys-loop receptors: new twists and turns. Trends Neurosci 27:329-336.

Mangin JM, Baloul M, Prado DC, Rogister B, Rigo JM, Legendre P (2003) Kinetic properties of the $\alpha 2$ homo-oligomeric glycine receptor impairs a proper synaptic functioning. J Physiol (Lond) 553:369-386.

Miyazawa A, Fujiyoshi Y, Stowell M, Unwin N (1999) Nicotinic acetylcholine receptor at $4.6 \AA$ resolution: transverse tunnels in the channel wall. J Mol Biol 288:765-786.

Miyazawa A, Fujiyoshi Y, Unwin N (2003) Structure and gating mechanism of the acetylcholine receptor pore. Nature 423:949-955.

Monod J, Wyman J, Changeux J-P (1965) On the nature of allosteric transitions: a plausible model. J Mol Biol 12:88-118.

Rees MI, Lewis TM, Kwok JB, Mortier GR, Govaert P, Snell RG, Schofield PR, Owen MJ (2002) Hyperekplexia associated with compound heterozygote mutations in the $\beta$-subunit of the human inhibitory glycine receptor (GLRB). Hum Mol Genet 11:853-860.

Sakmann B, Patlak J, Neher E (1980) Single acetylcholine-activated channels show burst-kinetics in presence of desensitizing concentrations of agonist. Nature 286:71-73.

Salamone FN, Zhou M, Auerbach A (1999) A re-examination of adult mouse nicotinic acetylcholine receptor channel activation kinetics. J Physiol (Lond) 516:315-330.

Self SG, Liang K-Y (1987) Asymptotic properties of maximum likelihood estimators and likelihood ratio tests under nonstandard conditions. J Am Stat Assoc 82:605-610.

Shan Q, Haddrill JL, Lynch JW (2001) Ivermectin, an unconventional agonist of the glycine receptor chloride channel. J Biol Chem 276:12556-12564.

Sine SM, Claudio T, Sigworth FJ (1990) Activation of Torpedo acetylcholine receptors expressed in mouse fibroblasts. Single channel current kinetics reveal distinct agonist binding affinities. J Gen Physiol 96:395-437.

Singer JH, Talley EM, Bayliss DA, Berger AJ (1998) Development of glycinergic synaptic transmission to rat brain stem motoneurons. J Neurophysiol 80:2608-2620.

Stoica P, Selén Y, Li J (2004) On information criteria and the generalised likelihood ratio test of model order selection. IEEE Signal Proc Lett 11:794-797.

Takahashi T, Momiyama A (1991) Single-channel currents underlying glycinergic inhibitory postsynaptic responses in spinal neurons. Neuron 7:965-969.

Takahashi T, Momiyama A, Hirai K, Hishinuma F, Akagi H (1992) Functional correlation of fetal and adult forms of glycine receptors with developmental changes in inhibitory synaptic receptor channels. Neuron 9:1155-1161.

Tong G, Jahr CE (1994) Block of glutamate transporters potentiates postsynaptic excitation. Neuron 13:1195-1203.

Unwin N (1995) Acetylcholine receptor channel imaged in the open state. Nature 373:37-43.

Unwin N, Miyazawa A, Li J, Fujiyoshi Y (2002) Activation of the nicotinic acetylcholine receptor involves a switch in conformation of the a subunits. J Mol Biol 319:1165-1176.

Wyllie DJA, Béhé P, Colquhoun D (1998) Single-channel activations and concentration jumps: comparison of recombinant NR1a/NR2A and NR1a/NR2D NMDA receptors. J Physiol (Lond) 510:1-18.

Wyman J, Allen DW (1951) The problem of the heme interactions in hemoglobin and the basis of the Bohr effect. J Polym Sci VII:499-518.

Wyman J, Gill SJ (1990) Binding and linkage functional chemistry of biological macromolecules. Mill Valley, CA: University Science Books. 